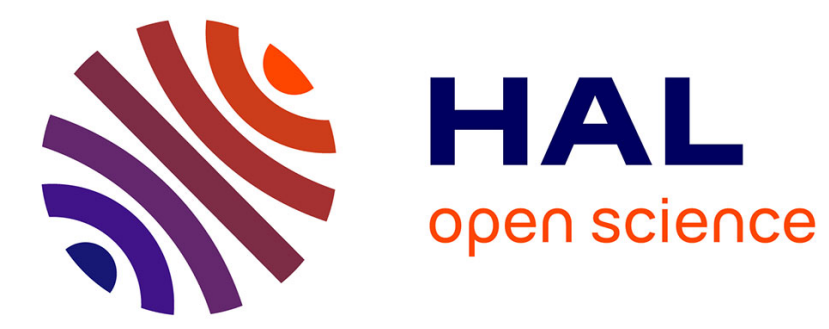

\title{
Acoustic modes in jet and wake stability
}

Eduardo Martini, André Cavalieri, Peter Jordan

\section{To cite this version:}

Eduardo Martini, André Cavalieri, Peter Jordan. Acoustic modes in jet and wake stability. Journal of Fluid Mechanics, 2019, 867, pp.804-834. 10.1017/jfm.2019.148 . hal-02351684

\section{HAL Id: hal-02351684 \\ https://hal.science/hal-02351684}

Submitted on 6 Nov 2019

HAL is a multi-disciplinary open access archive for the deposit and dissemination of scientific research documents, whether they are published or not. The documents may come from teaching and research institutions in France or abroad, or from public or private research centers.
L'archive ouverte pluridisciplinaire HAL, est destinée au dépôt et à la diffusion de documents scientifiques de niveau recherche, publiés ou non, émanant des établissements d'enseignement et de recherche français ou étrangers, des laboratoires publics ou privés. 


\title{
Acoustic modes in jet and wake stability
}

\author{
Eduardo Martini ${ }^{1} \dagger$, André V. G. Cavalieri ${ }^{1}$ and Peter Jordan ${ }^{2}$ \\ ${ }^{1}$ Instituto Tecnológico de Aeronáutica, São José dos Campos/SP - Brazil \\ ${ }^{2}$ Université de Poitiers, France \\ (Received xx; revised xx; accepted $\mathrm{xx}$ )
}

Motivated by recent studies that have revealed the existence of trapped acoustic waves in subsonic jets (Towne et al. J. Fluid Mech. vol 825, 2017, p. 1113-1152), we undertake a more general exploration of the physics associated with acoustic modes in jets and wakes, using a double vortex-sheet model. We show how jets and wakes may both behave as waveguides under certain conditions, emulating ducts with soft or hard walls, with the vortex-sheet impedance providing effective 'wall' conditions. We consider, in particular, the role that upstream-travelling acoustic modes play in the dispersion-relation saddle points that underpin the onset of absolute instability. The analysis illustrates how departure from duct-like behaviour is a necessary condition for absolute instability, and this provides a new perspective on the stabilising and destabilising effects of reverse flow, temperature ratio and compressibility; it also clarifies the differing symmetries of jet (symmetric) and wake (antisymmetric) instabilities. An energy balance, based on the vortex-sheet impedance, is used to determine stability conditions for the acoustic modes: these may become unstable in supersonic flow due to an energy influx through the shear layers. Finally, we construct the impulse response of flows with zero and finite shear-layer thickness. This allows us to show how the long-time wavepacket behaviour is indeed determined by interaction between Kelvin-Helmholtz and acoustic modes.

\section{Introduction}

Flow instabilities may be broadly divided into two categories, convective and global. The former involve disturbances that undergo spatial growth as they are convected away from their inception point. In such cases, all regions of the flow eventually return to their equilibrium state, once the disturbance has passed. In parallel or weakly non-parallel frameworks, the dynamics of such flows are underpinned by local, convectively unstable, downstream-travelling modes, such as the Kelvin-Helmholtz (KH) mode, typically observed in free shear layers, or Tollmien-Schlichting (TS) waves, typically observed in boundary layers. Globally unstable flows, on the other hand, involve disturbances that undergo temporal growth throughout the domain.

When analysed locally, global instability generally involves a feedback loop between upstream- and downstream-travelling modes. In free-shear flows, the downstreamtravelling mode is frequently a $\mathrm{KH}$ instability, whereas the nature of the upstreamtravelling mode may vary depending on the flow considered. Powell (1953) proposed that acoustic waves are responsible for the feedback that underpins tonal behaviour observed in underexpanded supersonic jets which screech, a concept later used to explain the tonal dynamics of impinging jets (Krothapalli 1985; Krothapalli et al. 1999; Gojon et al. 2015; Gojon \& Bogey 2017; Bogey \& Gojon 2017) and cavity flows (Karamcheti 1956; Rossiter 1964; Rowley et al. 2002; Yamouni et al. 2013). In these flows, upstream

$\dagger$ Email address for correspondence: emartini@ita.br 
disturbances are spatially amplified up to some downstream point at which they undergo an interaction (with a solid surface where impinging jets and cavities are concerned, with shock-cells in the case of screeching jets) that causes them to be scattered into, among other things, upstream-travelling sound waves, which are then reflected in the nozzle plane. When the phases of the upstream- and downstream-travelling waves are matched at the boundaries, and the reflection conditions are such that positive gain is possible, one has resonance, and global instability.

Towne et al. (2017) showed that, under certain conditions, discrete acoustic modes may be supported within the potential core of a jet, which behaves as a wave guide. Much of the flow physics could be understood using three simplified models: a cylindrical, soft-walled duct; a cylindrical vortex sheet; and a locally parallel, finite-thickness jet. Turning points associated with the narrowing of the potential core were identified at which the guided, propagative acoustic modes become evanescent, whence their reflection into upstream-travelling guided modes that are subsequently reflected in the nozzle plane. The same mechanism was revealed in a global-mode analysis (Schmidt et al. 2017), thus explaining the existence of weak tones observed in simulations and experiments (Brès et al. 2018).

To understand the correspondence between the soft-walled duct model and the vortexsheet and finite-thickness jet models, properties of the eigenmodes of the latter two were considered. It was thus shown how round jets can support phenomena such as refraction and total reflection (illustrated in figure 1), and how the latter may cause the jet to behave as a wave guide, for both upstream- and downstream-travelling, neutrally stable, acoustic disturbances. Any mechanism enabling interaction between the KH mode and the guided, upstream-travelling acoustic modes, may potentially lead to global instability. Jordan et al. (2018) show, for instance, how such interactions underpin tones that occur when a turbulent jet grazes a sharp edge.

Such long-range mechanisms are not the only path to global instability. Wakes (Noack \& Eckelmann 1994) and hot jets (Monkewitz \& Sohn 1988) exhibit global instability that is not related to wave reflections, but, rather, to flow features that can be studied in a locally parallel framework. Huerre \& Monkewitz (1985), adapting the works of Briggs (1964) and Bers (1975), provide a framework for the study of absolute instability in parallel base flows, showing how the long-time impulse response is dominated by an $\exp \left(-\mathrm{i} \omega_{0} t\right)$ time dependence, the absolute frequency, $\omega_{0}$, being obtained from a saddle point formed between upstream- and downstream-travelling branches of the dispersion relation. For slowly-diverging base flows, the analysis can be extended using asymptotic methods that show how a spatially localised pocket of absolute instability is a requirement for global instability (Chomaz et al. 1991; Monkewitz et al. 1993). A recent global stability analysis for slowly-diverging base flows, by Siconolfi et al. (2017), shows how accurate predictions of global modes may be obtained using the asymptotic approach.

While global stability problems may now be solved numerically (Theofilis 2011), knowledge of the local mechanisms is frequently necessary, both for a complete understanding of the underlying flow physics, and for the proposition of simplified models. The combination of local and global analyses was the key to understanding the resonant trapped waves in the study of Schmidt et al. (2017).

For both wakes (typically globally unstable) and jets (typically globally stable), the downstream-travelling mode is a $\mathrm{KH}$ wave. One may therefore infer that the contrast in stability characteristics of jets and wakes is due to the nature of their upstream-travelling modes, which are often left unexplored. Motivated by the work of Towne et al. (2017), we perform a systematic study of acoustic modes supported by jets and wakes, our ultimate goal being to clarify their role in global instability. We use a parallel double vortex-sheet 

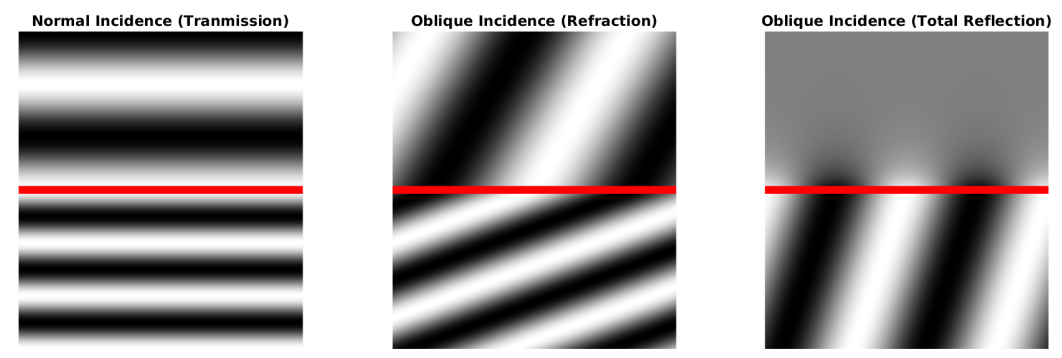

Figure 1: Illustration of transmission, refraction and total reflection of waves travelling between two media. Reflected waves are not shown in the interest of figure clarity.

(DVS) model to provide a general representation of both jets and wakes. We explore the conditions, for both flows, under which trapped acoustic modes may be found, we study the associated physical mechanisms, and, finally, we establish the role that these modes play in absolute instability.

While we focus primarily on the incompressible limit, where acoustic modes become evanescent pressure waves, we nonetheless consider certain aspects of the role of compressibility. Specifically, we show how the conditions for spatial stability of propagative acoustic modes in the DVS model are associated with their phase speeds: acoustic modes are neutrally stable when their phase-speed is subsonic with respect to the outer flow, and they may be stable or unstable when their phase-speed is supersonic, instability being shown to be associated with an influx of fluctuation energy through the vortex sheets. The unstable scenario is shown to correspond to that identified by Tam \& $\mathrm{Hu}(1989 b)$.

The paper is organised as follows. In $\S 2$ we present a double vortex-sheet (DVS) model. Section $\S 3$ is concerned with a demonstration of the existence of guided acoustic modes in the DVS and a consideration of the role they play in absolute instability. In Section $\S 4$ an energy balance approach is used to provide a more complete physical interpretation of the guided acoustic modes; effects of finite impedance are also considered, as is the relation of the guided modes to spatially unstable acoustic modes identified by Tam \& $\mathrm{Hu}(1989 b)$ in the supersonic regime. In Section $\S 5$ we construct the impulse responses of the DVS, and use it to explore compressibility and finite-thickness effects. A closing discussion is provided in $\S 6$.

\section{Double vortex-sheet (DVS) flow model}

We consider the compressible, linearised Euler equations for a double vortex sheet (DVS) (Lessen et al. 1965), whose base state is defined as,

$$
U(y), a(y), T(y), \rho(y)=\left\{\begin{array}{ll}
U_{\text {out }}, a_{\text {out }}, T_{\text {out }}, \rho_{\text {out }} & ,|y|>1 \\
U_{\text {in }}, a_{\text {in }}, T_{\text {in }}, \rho_{\text {in }} & ,|y|<1
\end{array},\right.
$$

where $U, a, T$ and $\rho$ denote, respectively, base-flow velocity, sound speed, temperature and density, subscripts in and out referring, respectively, to the inner $(|y|<1)$ and outer $(|y|>1)$ regions, as shown in figure 2. Assuming ideal gas relations, the following 


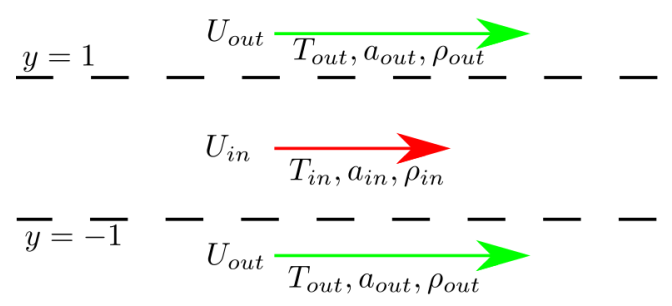

Figure 2: Double vortex-sheet (DVS) base flow.

parameterisation is obtained,

$$
\begin{array}{lccl}
U_{\text {in }}=-V_{\text {ref }} & T_{\text {in }}=1 & a_{\text {in }}=\frac{1}{M} & \rho_{\text {in }}=1 \\
U_{\text {out }}=1-V_{\text {ref }} & T_{\text {out }}=\frac{1}{T_{r}} & a_{\text {out }}=\frac{1}{M \sqrt{T}_{r}} & \rho_{\text {out }}=\rho_{r} T_{r}
\end{array} .
$$

This leads to the following non-dimensional parameters: the Mach number, $M$, the Strouhal number, $S t=\omega / \pi$, the inner-to-outer temperature and density ratios, respectively, $T_{r}$ and $\rho_{r}$. The parameterisation is such that the inner fluid properties and the shear strength remain constant. On varying $V_{\text {ref }}$ from 0 to 1 , the system transitions from an ideal wake to an ideal left-pointing jet, as illustrated in figure 3 . But $V_{\text {ref }}$ can also be considered to define the reference frame in which the flow is observed, thus clarifying a similarity between jets and wakes: they are underpinned by the same physical mechanisms, only observed from different perspectives. Because the choice to consider left-pointing jets, described by $V_{\text {ref }}=1$, differs from the more usual convention, according to which jets are considered as right-pointing, wavenumbers and phases speeds associated with jet configurations will be plotted as $-\alpha$ and $-c$ in order to facilitate comparison with similar figures found in the literature.

Four regimes can be identified: (1) $V_{\text {ref }}<0$, which defines a wake with co-flow; (2) $0<V_{\text {ref }}<1$, defining a wake with reverse flow in the inner region; (3) $V_{\text {ref }}=1$, which corresponds to a left pointing jet; and, (4) $V_{r e f}>1$, which gives a left-pointing jet with a flight effect. The regime $0<V_{\text {ref }}<1$ could of course be understood to correspond to a left-pointing jet with counter flow, highlighting the point made above, that differences between jets and wakes are largely due to the choice of reference frame. Also, the limit between "wake behavior" and "jet behavior" is arbitrary; we choose, therefore, to refer to $V_{\text {ref }}<0.5$ as the wake-like regime and $V_{\text {ref }}>0.5$ as the jet-like regime, and the terms Wake and Jet are chosen to indicate $V_{\text {ref }}=0$ and 1 , respectively.

The variables of equation 2.1 are used in all derivations, and those of equation 2.2 are considered for the study of jet and wake configurations. Small perturbations, $u^{\prime}(x, y, t), v^{\prime}(x, y, t)$ and $p^{\prime}(x, y, t)$, are superposed on the base flow, $u^{\prime}$ and $v^{\prime}$ denoting velocity fluctuations in streamwise, $x$, and vortex-sheet-normal $y$, directions, respectively, $t$ denoting time, and $p^{\prime}$ denoting pressure fluctuations. The base flow is parallel, and thus homogeneous in the $x$ - and $t$-directions, leading to the normal-mode Ansatz,

$$
p^{\prime}(x, y, t)=\hat{p}(y) \mathrm{e}^{\mathrm{i}(\alpha x-\omega t)},
$$

for the pressure, and equivalent expressions for the other flow variables.

The inviscid, compressible Euler equations are linearised for the inner and outer regions, giving, 


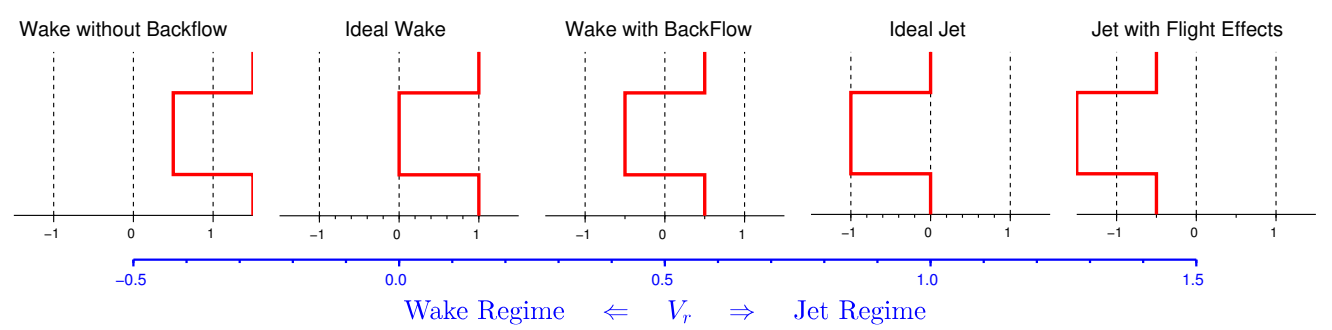

Figure 3: Illustration of the DVS parameterisation defined by equation 2.2 . The flow transitions between wake and jet behaviours with increasing $V_{\text {ref }}$.

$$
\begin{aligned}
& \left(\frac{\partial^{2}}{\partial y^{2}}+\frac{\left(\omega-U_{\text {in }} \alpha\right)^{2}}{a_{\text {in }}^{2}}-\alpha^{2}\right) \hat{p}_{\text {in }}=0, \\
& \left(\frac{\partial^{2}}{\partial y^{2}}+\frac{\left(\omega-U_{\text {out }} \alpha\right)^{2}}{a_{\text {out }}^{2}}-\alpha^{2}\right) \hat{p}_{\text {out }}=0,
\end{aligned}
$$

matching conditions between the domains giving,

$$
\begin{aligned}
\left(-\mathrm{i} \omega+\mathrm{i} U_{\text {in }} \alpha\right)^{2} \hat{\eta} & =\frac{1}{\rho_{\text {in }}} \frac{\partial \hat{p}_{\text {in }}}{\partial y}, \\
\left(-\mathrm{i} \omega+\mathrm{i} U_{\text {out }} \alpha\right)^{2} \hat{\eta} & =\frac{1}{\rho_{\text {out }}} \frac{\partial \hat{p}_{\text {out }}}{\partial y}, \\
\hat{p}_{\text {in }} & =\hat{p}_{\text {out }},
\end{aligned}
$$

where $\eta$ is the vortex-sheet displacement at $y= \pm 1$. The first two equations match interface displacements between the inner and outer regions, while the third imposes pressure continuity across the vortex-sheets. Additionally, it is required that $\hat{p}(y)$ be bounded at $\pm \infty$.

Solutions of the system are,

$$
\hat{p}_{s, a}(y)=\left\{\begin{array}{ll}
A_{s, a} \mathrm{e}^{-\xi_{\text {out }} y} & , y>1 \\
\mathrm{e}^{\xi_{\text {in }} y} \pm \mathrm{e}^{-\xi_{\text {in }} y} & ,|y|<1, \\
\pm A_{s, a} \mathrm{e}^{\xi_{\text {out }} y} & , y<-1
\end{array} \quad A_{s, a}=\frac{\mathrm{e}^{\xi_{\text {in }}} \pm \mathrm{e}^{-\xi_{\text {in }}}}{\mathrm{e}^{-\xi_{\text {out }}}}\right.
$$

with plus and minus signs corresponding to symmetric $(s)$ and anti-symmetric $(a)$ perturbations, where,

$$
\xi_{\text {in }, \text { out }}=\sqrt{\alpha^{2}\left(1-\left(\frac{U_{\text {in }, \text { out }}-c}{a_{\text {in }, \text { out }}}\right)^{2}\right)}
$$

and $c=\omega / \alpha$ is the streamwise phase velocity. The branch cut of equation 2.10 is chosen such that $-\pi / 2 \leqslant \arg \left(\xi_{\text {out }, \text { in }}\right)<\pi / 2$, as discussed by Tam \& Hu (1989b). The dispersion relations for the symmetric and antisymmetric modes are, respectively,

$$
\tanh \left(\xi_{\text {in }}\right)=-\frac{\rho_{\text {in }}}{\rho_{\text {out }}} \frac{\xi_{\text {out }}}{\xi_{\text {in }}}\left(\frac{U_{\text {in }}-c}{U_{\text {out }}-c}\right)^{2}, \quad \operatorname{coth}\left(\xi_{\text {in }}\right)=-\frac{\rho_{\text {in }}}{\rho_{\text {out }}} \frac{\xi_{\text {out }}}{\xi_{\text {in }}}\left(\frac{U_{\text {in }}-c}{U_{\text {out }}-c}\right)^{2},
$$


and the other flow variables can be recovered from the pressure distribution as,

$$
\hat{u}=\frac{\hat{p}}{(c-U) \rho}, \quad \hat{v}=\frac{\frac{\partial \hat{p}}{\partial y}}{\mathrm{i} \alpha(c-U) \rho}, \quad \hat{\rho}=\frac{\hat{p}}{a^{2}} .
$$

The singularities at $c=U_{\text {in }, \text { out }}$ are avoided by investigating only nearby points, $c \approx$ $\left.U_{\text {in,out }}\right)$.

\section{Acoustic modes and stability of the DVS flow}

Our goal is to explore the role of acoustic phenomena in the stability of jets and wakes, considered via the DVS model described in the previous section. What we refer to as acoustic modes are solutions of the convected wave equation,

$$
\frac{1}{a^{2}}\left(\frac{d}{d t}+U \frac{d}{d x}\right)^{2} p(x, y, t)-\nabla^{2} p(x, y, t)=0 .
$$

Note that for low frequency, and particularly in ducts (which we will show support dynamics analogous to those of the DVS), solutions of the wave equation are evanescent waves. In the incompressible limit, as $a \rightarrow \infty$, the cut-on frequency that marks transition between evanescent and propagative waves also goes to $\infty$. The evanescent waves that solve the wave equation in the incompressible limit are therefore synonymous with evanescent-wave solutions that exist for finite $a$, justifying the denomination 'acoustic' in reference to solutions in the incompressible limit, which we consider in what follows. For ducts with uniform flow, $U$, a mode with radial wavenumber, $n$, leads to dispersion relation and group-velocity definitions,

$$
\begin{aligned}
\alpha & =\frac{-U \omega \pm a \sqrt{\omega^{2}-(n \pi / 2)^{2}\left(a^{2}-U^{2}\right)}}{a^{2}-U^{2}}, \\
v_{\text {group }}=\frac{\partial \omega}{\partial \alpha} & =U+\frac{a}{(c-U)} a,
\end{aligned}
$$

which will be used throughout the work. Their derivation, along with an overview of duct acoustics, can be found in Appendix A.

\subsection{Incompressible regime}

We now consider the DVS flow in the incompressible limit. We show, firstly, that KH and acoustic duct-modes are asymptotic solutions of the DVS model. We then determine conditions for the existence of acoustic modes in terms of vortex-sheet impedances. This allows us to show how the vortex sheet (VS) may behave in the manner of a hard- or a soft-walled duct, depending on the region of the parameter space that is considered,

In the incompressible limit, equation 2.10 reduces to $\xi_{\text {in }, \text { out }}=\sqrt{\alpha^{2}}$. For $\left|a_{r}\right| \gg 1$, solutions of the dispersion relation given by 2.11 are KH modes (Lamb 1945, page 373),

$$
c=\frac{U_{\text {out }} \rho_{\text {out }}+U_{\text {in }} \rho_{\text {in }}}{\rho_{\text {out }}+\rho_{\text {in }}} \pm \mathrm{i} \frac{\sqrt{\rho_{\text {in }} \rho_{\text {out }}}}{\rho_{\text {out }}+\rho_{\text {in }}}\left(U_{\text {out }}-U_{\text {in }}\right),
$$

which may be symmetric or antisymmetric, as illustrated in figures 4 (a) and (b). Another asymptotic behaviour is obtained when $c \approx U_{\text {in }}$ or $c \approx U_{\text {out }}$ :

$$
\left.\begin{array}{l}
\text { for } c \approx U_{\text {in }} \quad: \tanh \xi_{\text {in }}=0 \quad \begin{array}{l}
\text { and } \\
\text { for } c \approx U_{\text {out }}
\end{array} \quad: \tanh \xi_{\text {in }}=\infty \\
\text { and } \xi_{\text {in }}=0 \\
\operatorname{coth} \xi_{\text {in }}=\infty
\end{array}\right\} \Rightarrow \xi_{\text {in }}=-\mathrm{i} \pi \frac{n}{2} .
$$




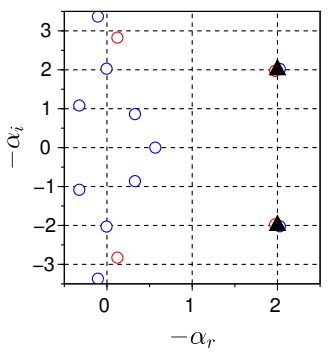

(a) Incompressible Jet, $\omega=2.5$
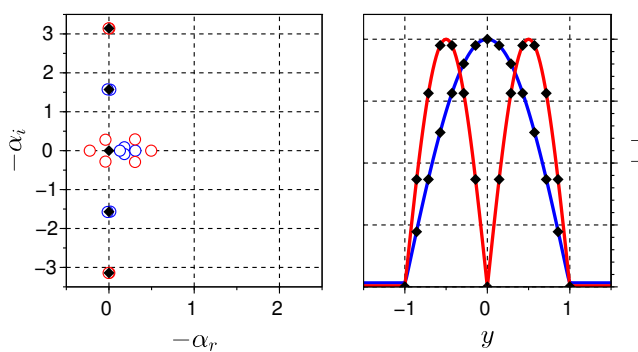

(c) Incompressible Jet, $\omega=0.2$
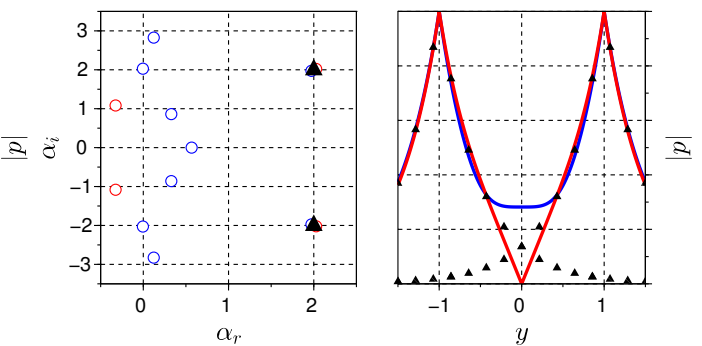

(b) Incompressible Wake, $\omega=2.5$
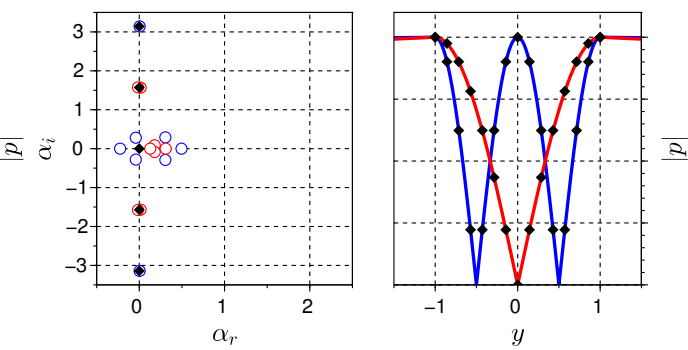

(d) Incompressible Wake, $\omega=0.2$

Figure 4: Comparison between DVS and KH modes (top) and duct-like modes (bottom). Spatial spectra are shown on the left of each sub plot, with blue and red circles indicating symmetric and antisymmetric DVS modes, respectively, black diamonds acoustic-duct modes and triangles single vortex-sheet $\mathrm{KH}$ modes. The right-hand sub plots show eigenmode comparisons between DVS (solid lines), acoustic-duct modes (diamonds) and single vortex-sheet $\mathrm{KH}$ modes (triangles).

Solving for $\alpha$ leads to,

$$
\alpha^{ \pm}=\frac{-U_{i n} \omega \pm a_{i n} \sqrt{\omega^{2}-\left(\pi \frac{n}{2}\right)^{2}\left(a_{i n}^{2}-U_{i n}^{2}\right)}}{a_{i n}^{2}-U_{i n}^{2}},
$$

which is the dispersion relation for the acoustics of hard- and soft-walled ducts (equation 3.2). For $U_{i n}=0, \alpha^{+}$and $\alpha^{-}$correspond to left- and right-travelling modes, but if a mean flow is present, the phase and/or group velocities of certain modes may change direction, as discussed in Appendix A. Figures 4 (c) and (d) show the similarity between acoustic modes in ducts and those of the DVS jet and wake. When $\omega \approx 0$ we have $c=\omega / \alpha \approx 0$ and so, $c \approx U_{\text {in }}$ and $c \approx U_{\text {out }}$, for the wake and jet, respectively, everywhere in the complex $\alpha$ plane, except near $\alpha=0$.

Motivated by the realisation that soft- and hard-walled duct-like behaviours reflect the vortex-sheet "stiffness", we perform a systematic study of the vortex-sheet impedance, $\mathcal{Z}_{V S}$, which will also be useful in identifying total-reflection conditions, as in Towne et al. (2017), when we consider compressible flows. The impedance of a single vortex-sheet, located at $y=1$, is calculated. Flows above and below the vortex sheet have properties of the outer and inner flows, respectively. For $y<1$ we assume $p^{\prime}(x, y, t)=A \hat{p}(y) \mathrm{e}^{\mathrm{i} \alpha x-\mathrm{i} \omega t}$, while for $y>1$ we enforce the Euler equations, $p^{\prime}(x, y, t)=B \mathrm{e}^{-\xi_{\text {out }} y+\mathrm{i} \alpha x-\mathrm{i} \omega t}$. The two domains are connected with interface conditions identical to those of equations 2.6-2.8, 
giving,

$$
\mathcal{Z}_{V S}(\omega, \alpha)=\left.\frac{p^{\prime}}{v^{\prime}}\right|_{y=1^{-}}=\rho_{\text {out }} \frac{\alpha}{i \xi_{\text {out }}} \frac{\left(c-U_{\text {out }}\right)^{2}}{c-U_{\text {in }}} .
$$

The impedance of the inner region, $\mathcal{Z}_{i n}$, is obtained from the $|y|<1$ solutions of equation 2.9 , for symmetric and antisymmetric modes, respectively,

$$
\mathcal{Z}_{i n, s}(\omega, \alpha)=-\rho_{i n} \alpha \frac{\left(c-U_{i n}\right)}{i \xi_{i n}} \tanh \left(\xi_{i n}\right), \quad \mathcal{Z}_{i n, a}(\omega, \alpha)=-\rho_{i n} \alpha \frac{\left(c-U_{i n}\right)}{i \xi_{i n}} \operatorname{coth}\left(\xi_{i n}\right)
$$

Note that the DVS dispersion relation (equation 2.11) is a compatibility condition between the impedances of the vortex-sheet and the inner flow region.

In order to determine whether the vortex-sheet impedance should be considered high or low, a reference impedance is defined. Assuming hard- or soft-walled duct modes $\left(\xi_{i n}=-\mathrm{i} n \pi / 2\right)$, the hyperbolic functions in the dispersion relation become either 0 or $\infty$; we thus use the other terms of the dispersion relation to define a reference impedance such that,

$$
\frac{\rho_{\text {in }}}{\rho_{\text {out }}} \frac{\xi_{\text {in }}}{\xi_{\text {out }}}\left(\frac{U_{\text {in }}-c}{U_{\text {out }}-c}\right)^{2}=\frac{\mathcal{Z}_{V S}}{\mathcal{Z}_{\text {ref }}} \approx\left\{\begin{array}{ll}
0 & , \text { with a soft-duct approximation } \\
\infty & , \text { with a hard-duct approximation }
\end{array},\right.
$$

where,

$$
\mathcal{Z}_{\text {ref }}(\omega, \alpha)=\rho_{\text {in }} \frac{\alpha}{\mathrm{i} \xi_{\text {in }}}\left(c-U_{\text {in }}\right) .
$$

The reference impedance depends only on the parameters of the inner region, and thus may be interpreted as an impedance to perturbations in the inner region, to which the vortex-sheet "stiffness" is compared. We are interested in the conditions under which the duct-mode Ansatz is self-consistent. A duct-mode Ansatz for the DVS is assumed, and the impedance ratio calculated; impedances of 0 and $\infty$ indicate self-consistency of the Ansatz; large (respectively small), but finite, values indicate that hard- (respectively soft-) walled duct modes are a good approximation for DVS modes

Impedance ratios are shown, as a function of $V_{\text {ref }}$ and $\omega$, in figure 5. A clear distinction is manifest between jet and wake behaviours at low frequency, low and high values of impedance-ratio being observed, respectively, in the jet $\left(V_{\text {ref }}=1\right)$ and wake $\left(V_{\text {ref }}=0\right)$ regimes, satisfying soft- and hard-walled acoustic duct Ansätze, respectively. At higher frequency the self-consistency condition is weakened, implying a departure from ductlike behaviour, which is manifest in the higher-frequency eigenfunctions of figure 6 (cf. DVS mode at $\omega=0.8$ for example): when the impedance-ratio values depart from those consistent with the Ansatz, the DVS eigenfunctions no longer match, but nonetheless resemble, duct modes, modified to have non-zero support in the outer flow. We may infer that the associated mechanisms are of an acoustic nature, but modified by interaction with the vortex sheets.

We note that the first harmonic duct-like modes of the jet and wake DVS solutions exhibit symmetries similar to those of unstable modes in hot jets (symmetric) and wakes (antisymmetric). This suggests that it is the duct-like modes that provide the upstreamtravelling dynamics necessary for absolute instability. The relation between acoustic modes and absolute instability is further explored in what follows. 

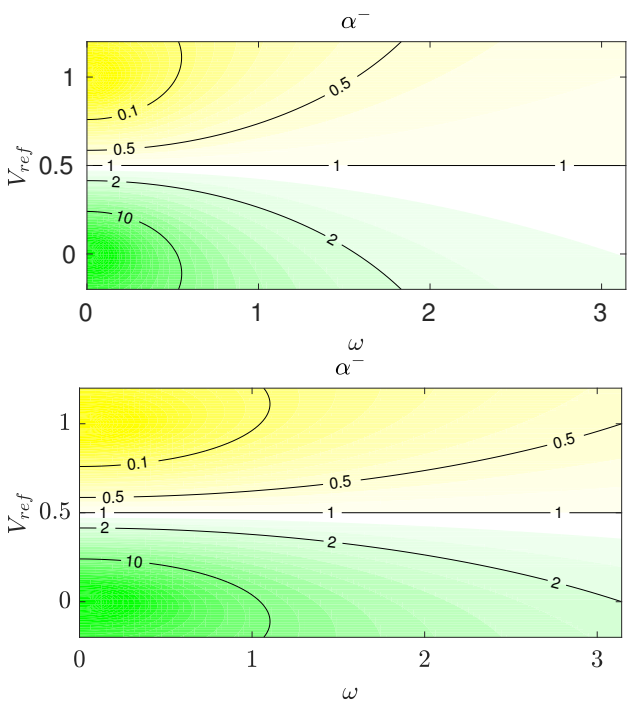
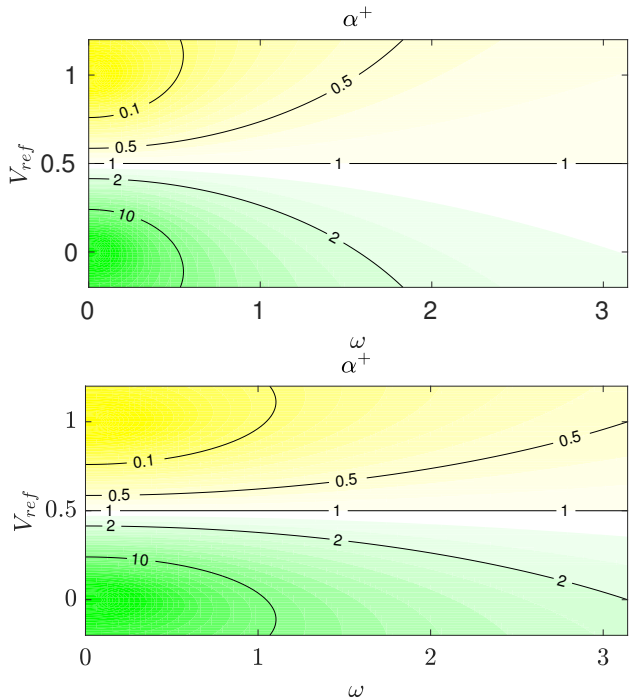

Figure 5: Impedance-ratio map indicating regions where modes resemble those of soft- (yellow) or hard-walled (green) acoustic ducts. A clear trend is identified at low frequency, wakes $\left(V_{\text {ref }}<0.5\right)$ and jets $\left(V_{\text {ref }}>0.5\right)$ exhibiting hard- and soft-walled behaviours, respectively. The distinction deteriorates at higher frequencies. The colour scale corresponds to absolute value of the impedance ratio $\left(\mathcal{Z}_{V S} / \mathcal{Z}_{r e f}\right)$ for $T_{r}=1, \rho_{r}=$ $1, M=0.01$. Top and bottom plots correspond, respectively, to first $(n=1)$ and second $(n=2)$ harmonic, duct-like modes.
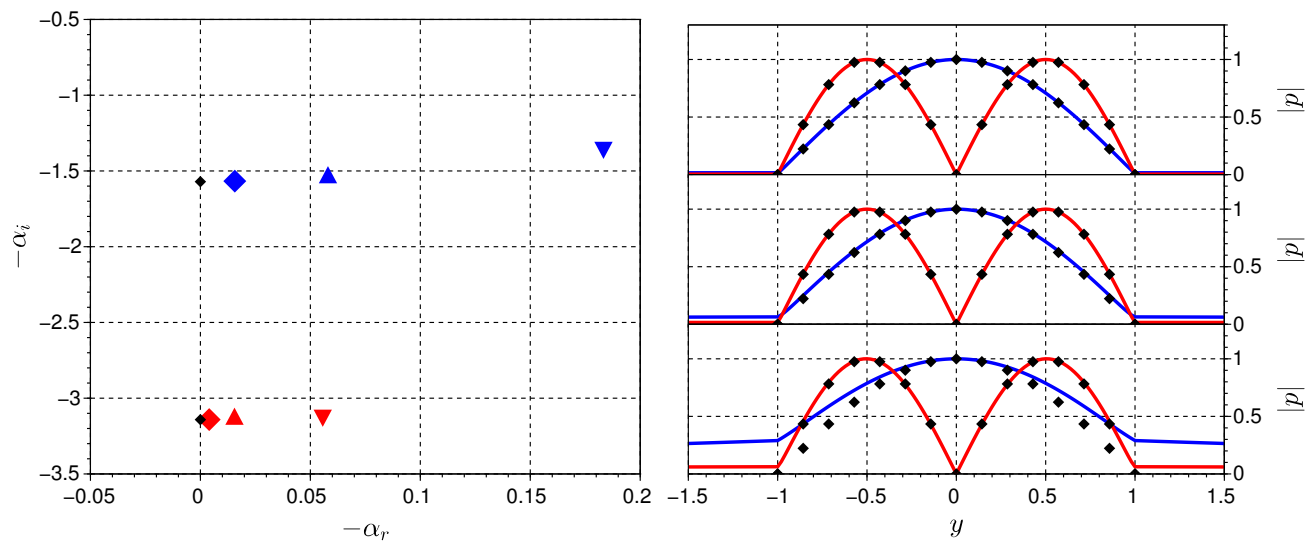

Figure 6: Deviation of DVS jet $\left(V_{\text {ref }}=1\right)$ modes from acoustic, duct-like behaviour with increasing frequency. Left plot shows DVS, the markers $\diamond, \triangle, \nabla$ indicating, respectively $\omega=0.2,0.4$ and 0.8 ; the right plot shows corresponding pressure eigenfunctions, for, from top to bottom, $\omega=0.2,0.4$ and 0.8 , black diamonds indicating the corresponding acoustic-duct mode.

\subsection{Absolute instability of incompressible wakes and jets}

We now wish to establish the relationship between the acoustic duct-like behaviour considered above and the absolute instability of jets and wakes. It is of course clear that, at the DVS saddle point that determines absolute instability, the DVS duct-like 

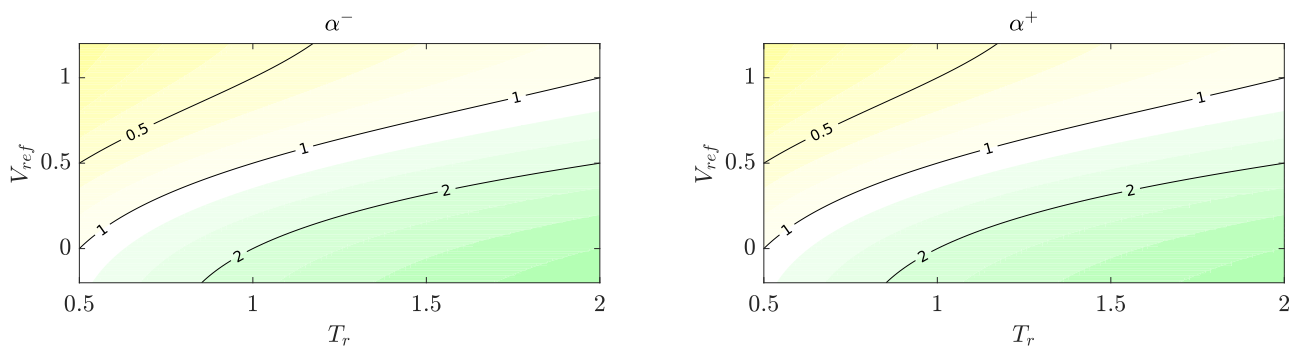

Figure 7: Impedance-ratio $\left(\mathcal{Z}_{V S} / \mathcal{Z}_{\text {ref }}\right)$ map showing dependence on $T_{r}$ and $V_{\text {ref }}$. Colour map is the same as in Figure 5, for $\rho_{r}=1, \omega=\pi / 2, M=0.01$, for the first harmonic duct mode $(n=1)$.

modes cannot exactly match true duct modes, as such saddle points do not exits in ducts. Indeed, the results to follow support the idea that a departure from duct-like behaviour is a necessary condition for the onset of absolute instability. We begin with a consideration of the destabilising effect of temperature, when jets are heated and wakes are cool (Yu \& Monkewitz 1990). Figure 7 shows the effect of $T_{r}$ on the impedance-ratio. This increases with increasing $T_{r}$, but with opposite effects for wakes and jets. When the wake is heated, conditions for duct-like behaviour are improved; when the jet is heated, on the other hand, the duct-like behaviour is degraded. This is a direct consequence of the different duct-behaviour approximations: duct-like behaviour in jets is soft-walled, whereas in wakes it is hard-walled.

We now consider the saddle point that marks the transition between convective and absolute instability. As established by Huerre \& Monkewitz (1990) for parallel flows, following Briggs (1964) and Bers (1975), the distinction between absolute and convective instability is given by the imaginary part of the frequency associated with a double root, $\alpha_{0}$, of the spatial stability problem, such that $\partial \omega / \partial \alpha\left(\alpha_{0}\right)=0$. The double root must be formed between two modes, one of which is downstream-travelling, the other being upstream-travelling. Absolute instability can be understood as due to a resonant interaction between modes of opposite group velocities; there is disturbance growth if $\operatorname{Im}\left(\omega_{0}\right)>0$, where $\omega_{0}=\omega\left(\alpha_{0}\right)$. It is known that vortex-sheet models are ill-posed, in so far as they present unbounded growth with increasing wavenumber and/or frequency, which precludes calculation of the impulse response. We may nonetheless compute the saddle point, as this permits a useful discussion of the role of the duct-like modes in absolute instability, and we leave discussion the effects of these on the impulse respons to Section $§ 5.2$.

Isothermal DVS jets and wakes are convectively unstable in the absence of co-flow. By varying $T_{r}$ and mapping loci of the roots for real frequencies a "pinching" between two branches is observed, indicating a saddle-point leaving the negative $\omega_{i}$ plane, and signaling the onset of absolute instability. The neutral saddle point for the jet is observed at $T_{r} \approx 1.26$, whereas for the wake it is found at $T_{r} \approx 0.79$, as shown in figure 8 . The branches associated with the saddle point can be recognised as the acoustic and $\mathrm{KH}$ branches; this confirms that the acoustic modes underpin absolute instability. Figures 7 and 8 show how destabilisation is associated with a deterioration of the duct-like behaviour. Equivalent results are obtained when the effect of backflow is considered, as can be seen in figures 5 and 9 .

We see how a departure from duct-like behaviour seems to be a necessary for interaction between $\mathrm{KH}$ and acoustic modes, and therefore for absolute instability. The $\mathrm{KH}$ and 

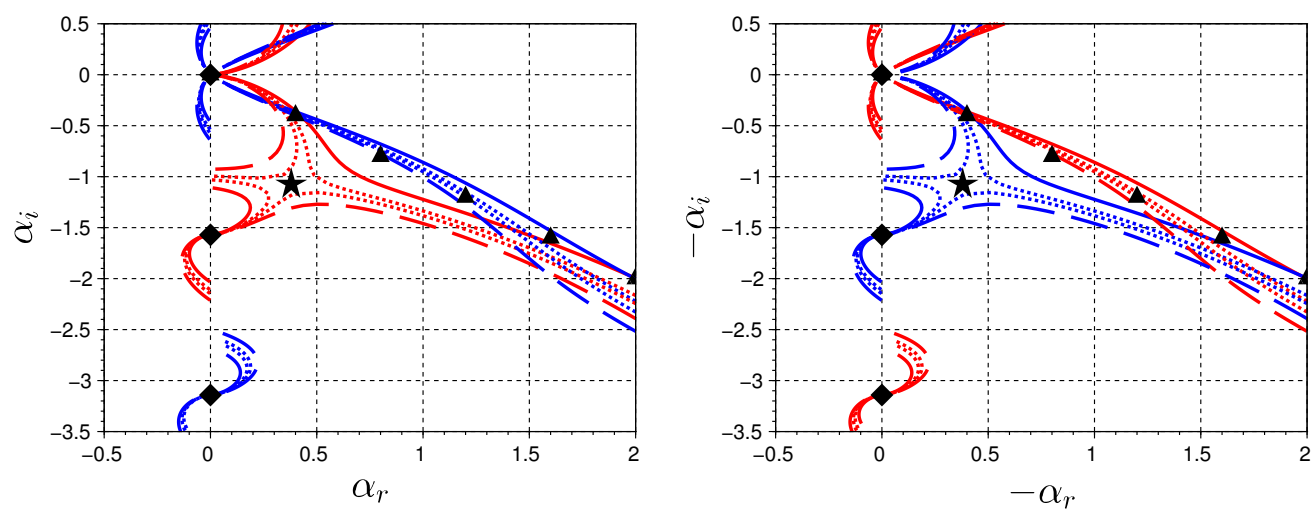

Figure 8: Spatial eigenspectra for incompressible $(M=0.01)$ wake (left) and jet (right), showing "pinching" of the acoustic and KH branches with temperature variation. Solid, dotted, and dashed lines corresponds to $T_{r}$ of $(1.00,0.83,0.76,0.66)$ on the left and $(1.0,1.2,1,3,1.5)$ on the right. Blue/red lines indicate antisymmetric/symmetric modes, diamonds/triangles indicate duct/KH modes. Neutral saddles location with real $\omega_{0}$ are found at $T_{r} \approx 1.26$ for the jet and at $T_{r} \approx 0.79$ for the wake (black stars). Loci are shown for $\omega \in[0,1.5 \pi]$.
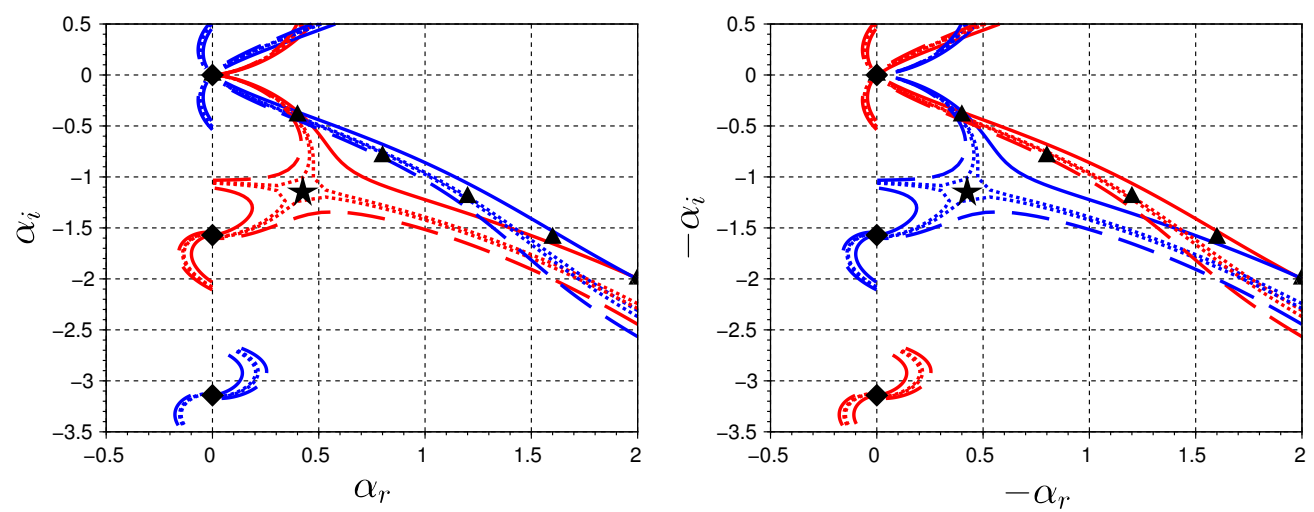

Figure 9: Same as Figure 8, but with saddle point formed due to changes in $V_{\text {ref }}$. Solid, dotted, and dashed lines corresponds to $V_{\text {ref }}=(1.00,0.95,0.93,0.90)$ for the jet and $(0.00,0.06,0.07,0.10)$ for the wake. Neutral saddles (black stars), are found for $V_{\text {ref }}=$ 0.935 and 0.065 for the jet and wake, respectively.

acoustic branches must move away from their asymptotic loci in order to meet at the saddle point, and with this movement, their eigenfunctions are deformed with respect to their asymptotic forms. This provides the following interesting interpretation of saddlepoint ringing: deterioration of the asymptotic duct-mode form involves an extension of the acoustic mode support across the vortex-sheet and into the outer flow, a "reaching outward", while the asymptotic KH deterioration involves a "reaching inward" of the KH mode. The process of absolute instability involves the modes reaching radially towards one another in order to interact. 

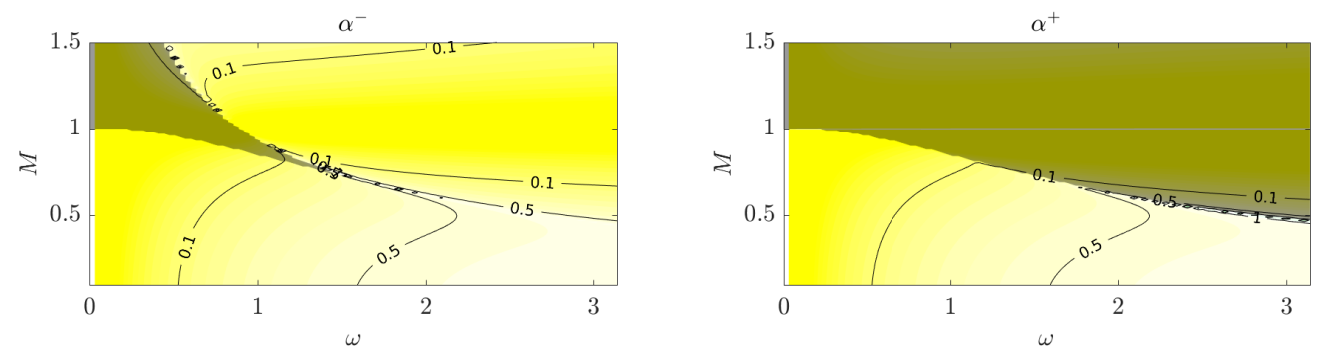

(a) Jet $\left(V_{r e f}=1\right)$
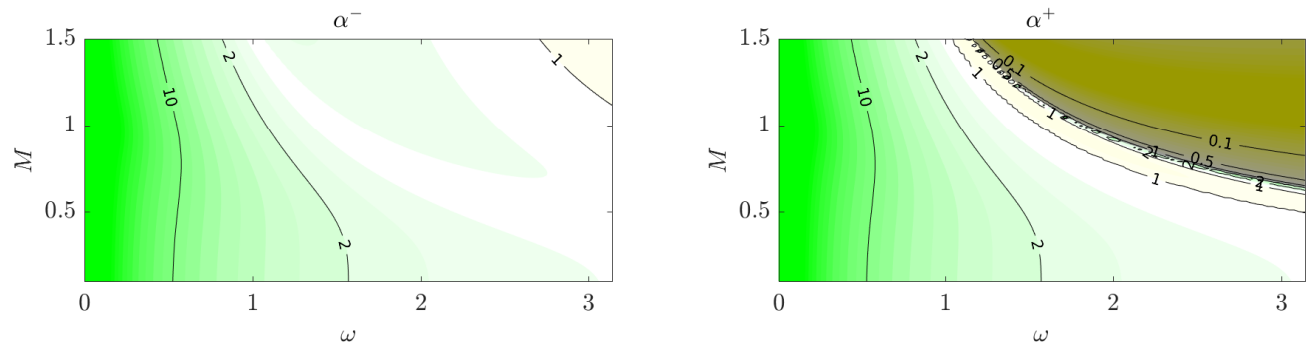

(b) Wake $\left(V_{\text {ref }}=0\right)$

Figure 10: Impedance-ratio $\left(\mathcal{Z}_{V S} / \mathcal{Z}_{\text {ref }}\right)$ map showing dependence on $\omega$ and $M$. For the jet, both $\alpha^{+}$and $\alpha^{-}$modes exhibit total reflection (shaded areas), while for the wake this is only the case for $\alpha^{+}$(downstream-traveling) modes. The colour map is the same as in 5 , for $T_{r}=1, \rho_{r}=1, \omega=\pi / 2$, and the first duct mode $(n=1)$.

\subsection{Acoustic modes in the compressible regime}

We have seen, in the previous section, how a resonance between $\mathrm{KH}$ and acoustic modes underpins the transition to absolute instability in the incompressible regime, under the effects of both $V_{\text {ref }}$ and $T_{r}$. We now explore some aspects of the compressible regime, where richer dynamics can occur thanks to the existence of propagative waves, both inside and outside the vortex-sheet, and the internal trapping of these, as found by Towne et al. (2017) and Schmidt et al. (2017).

The self-consistency impedance condition is extended to incorporate total internal reflection, which also leads to duct-like behaviour. Equation 3.7 shows how, for propagative modes (real $\alpha$ and $\omega$ ), the impedance is purely imaginary only if $\xi_{\text {out }}$ is real, which occurs when $\left|c-U_{\text {out }}\right|<a_{\text {out }}$; i.e. when the mode is subsonic, and thus evanescent, in the outer region. As the real part of the impedance is associated with energy flux through the boundary (Rienstra \& Hirschberg 2018), a purely imaginary impedance implies zero energy flux, due to total internal reflection and an internal trapping of fluctuation energy. A broader discussion of the relationship between impedance and energy flux is provided in section 4.

Figure 10 shows impedance ratios for isothermal jets and wakes. In the shaded regions the impedance is purely imaginary. For a wake, subsonic phase velocity with respect to the outer stream ( $c$ between $1 \pm a_{\text {out }}$ ) is only observed for the downstream-travelling $\left(\alpha^{+}\right)$ mode. For the jet, due to the presence of inner flow, both $\alpha^{+}$and $\alpha^{-}$modes may have subsonic phase velocity ( $c$ between $\pm a_{\text {out }}$ ). This distinction between the jet and the wake suggests that compressibility may have a greater stabilising effect in jets, which will be confirmed in $§ 5.1$, where we consider the impulse response of the DVS. 


\subsection{Summary}

We have shown how duct-like behaviour is associated with extreme values of vortexsheet impedance, and how the saddle point underpinning absolute instability is formed by $\mathrm{KH}$ and upstream-travelling acoustic branches when the conditions for asymptotic $\mathrm{KH}$ and duct-like behaviours are deteriorated. This is consistent with known destabilisation trends associated with temperature-ratio, co-flow and compressibility effects, and, furthermore, it clarifies the different symmetries of unstable jet and wake modes. The analysis confirms the essential role played by upstream-travelling acoustic modes in the instability of jets and wakes.

\section{Energy balance}

We now consider the implications of finite VS impedance. This is done by performing an energy-flux analysis, which also allows us to develop a more complete physical interpretation of the vortex-sheet behavior. A similar procedure to that applied in $\S 3.1$ is used: duct-mode Ansätze are assumed and corresponding impedances and energy fluxes are calculated.

The energy conservation law reads, (Goldstein 1976; Pierce 1981),

$$
\frac{\partial E}{\partial t}+\nabla \cdot \boldsymbol{J}=0
$$

where $E$ is the total energy and $\boldsymbol{J}$ the energy flux. This expression for energy conservation is only valid for small perturbations in uniform flows, and thus will only be applied to control volumes localised in the inner or outer regions. Assuming a stationary regime, where mean energy density is constant in time, equation 4.1 becomes $\langle\nabla \cdot \mathbf{J}\rangle=0$, where $\langle\cdot\rangle$ denotes a time average. The flux is given by,

$$
\boldsymbol{J}=\left(\frac{a^{2} \rho^{\prime}}{\rho}+\boldsymbol{u}^{\prime} \cdot \boldsymbol{U}\right)\left(\rho \boldsymbol{u}^{\prime}+\rho^{\prime} \boldsymbol{U}\right),
$$

which for the no-flow case can be readily identified with the acoustic intensity $p^{\prime} \boldsymbol{u}^{\prime}$. Assuming propagative acoustic modes, $\boldsymbol{J}$ components can be written, using equations 2.12 , as,

$$
\begin{aligned}
& J_{i}(x, y, t)=\left(\Re\left(\hat{p}(y) \mathrm{e}^{\mathrm{i}(\alpha x-\omega t)}\right)\right)^{2} \frac{1}{\rho}\left(\frac{1}{c-U}+\frac{U}{a^{2}}\right)\left(\frac{c}{c-U}\right), \\
& J_{j}(x, y, t)=\Re\left(\hat{v}(y) \mathrm{e}^{\mathrm{i}(\alpha x-\omega t)}\right) \Re\left(\hat{p}(y) \mathrm{e}^{\mathrm{i}(\alpha x-\omega t)}\right)\left(\frac{c}{c-U}\right) .
\end{aligned}
$$

The energy balance will be considered in two different manners: (1) a comparison of the magnitude of $\boldsymbol{J}$ in the inner and outer regions will be used to justify soft- and hardwalled duct approximations when $c \approx U_{\text {out }}$ and $c \approx U_{\text {in }} ;(2)$ a comparison of the energy flux in cross sections of the inner region and fluxes across the vortex-sheets will be used to derive corrections for cases where the impedance is finite.

\subsection{Soft- and hard-walled approximations}

As we have seen, small and large impedance ratios imply modes that behave as in a duct with uniform flow. In $\$ 3.1$ these limits were found when $c \approx U_{\text {out }}$ or $c \approx$ $U_{i n}$. The energy density flux relations defined by equations $4.2-4.4$ can be used to clarify the underlying physical mechanisms. The energy flux (equation 4.2) comprises scalar and vector components, in the first and second parentheses, respectively. The 


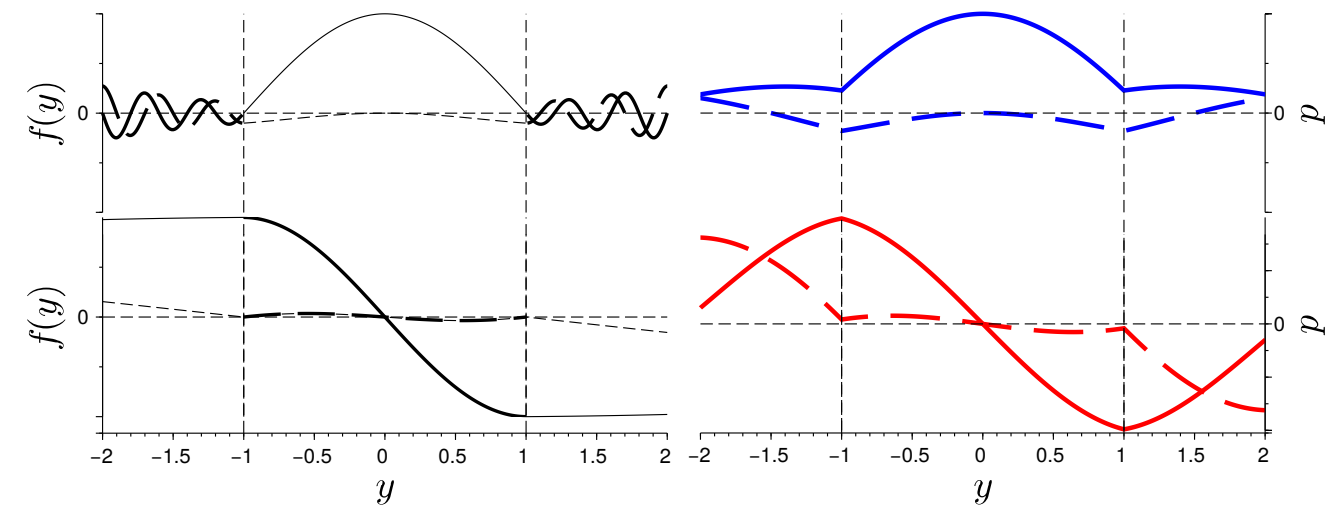

Figure 11: Analogy with vibration of an infinite string with DVS modes exhibiting discontinuous density and pressure. The left plot shows an infinite, vibrating string with discontinuous density, the thick line indicating higher density. The right plots show ductlike modes of the jet (top) and the wake (bottom) for $\omega=0.8$. Solid and dashed lines indicate the real and imaginary parts of the eigenfunctions, respectively.

former is proportional to $c /(c-U)$ and leads to high magnitude of $\boldsymbol{J}$ when $c \approx U$. Pressure continuity requires that the amplitude of the transmitted wave equal the sum of incident- and reflected-wave amplitudes. When $c \approx U_{\text {out }}$, the incident wave has a high energy density, and phase-opposition of the reflected wave reduces the amplitude of the transmitted wave.

A direct analogy may be drawn with the following simplified model: a wave travelling on a stretched string meets a discontinuity in string density, with the string becoming suddenly much heavier. Here also, reflected waves limit the amplitude at the string discontinuity, which behaves as a rigid constraint. This is analogous to the soft-wall condition, string displacement playing the role of acoustic pressure in the DVS. Similarly, the case where $c \approx U_{i n}$ is analogous to a string discontinuity at which the string becomes much lighter. In-phase reflection limits stresses at the discontinuity: a forcerelieving boundary condition exists, and outgoing waves, travelling on the light string, have insignificant energy content, and thus only slightly damp oscillations of the heavy string. These analogies are illustrated in figure 11, and the corresponding formulations are provided in Appendix C.

\subsection{Energy balance in the inner region}

The effect of finite impedance can be explored by energy balance analysis of the control volume shown in figure 12. Specifically, we compare energy fluxes across sections of the inner region $(F)$ to fluxes to and from the interfaces $\left(J_{v s}\right)$. The inner flux is given by,

$$
F(x, t)=\int_{-1}^{1} J_{i}(x, y, t) d y,
$$

while energy flux at the upper and lower vortex-sheets is given by,

$$
J_{v s}(x, t)=J_{j}(x, 1, t)-J_{j}(x,-1, t),
$$

where $\left(J_{i}, J_{j}\right)$ are the components of $\boldsymbol{J}$, as defined by equation 4.2.

The time-averaged energy-conservation law for the control volume becomes, 


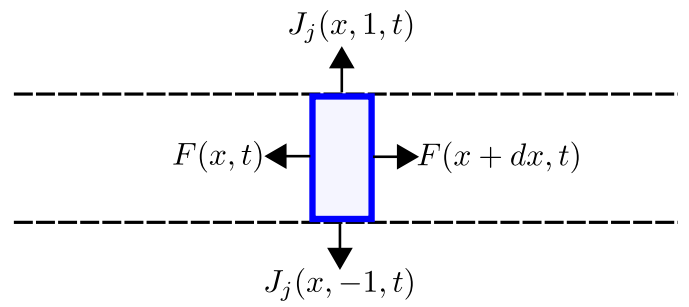

Figure 12: Schematic depiction of energy balance analysis. Control volume shown in blue, vortex-sheets indicated by the black dashed lines and energy fluxes by arrows.

$$
\frac{\partial}{\partial x} \mathcal{F}(x)=-\mathcal{J}_{j}(x)
$$

with

$$
\mathcal{F}(x)=\langle F(x, t)\rangle, \quad \mathcal{J}_{j}(x)=\left\langle J_{v s}(x, t)\right\rangle .
$$

Leading-order approximations of energy fluxes in the inner region and at the interfaces are obtained by assuming hard- and soft-walled duct behaviour,

$$
\hat{p}(y)=A\left(\mathrm{e}^{\xi_{\text {in }} y} \pm \mathrm{e}^{-\xi_{\text {in }} y}\right),
$$

the plus and minus signs being associated with symmetric and anti-symmetric modes, respectively. The time-averaged duct-like energy flux for the symmetric mode is,

$$
\begin{aligned}
\mathcal{F}(x)=\langle F(x, t)\rangle & =\int_{-1}^{1}\left\langle\boldsymbol{J}_{i}(y)\right\rangle d y \\
& =\frac{|A|^{2}}{\rho_{i n}} \frac{c}{c-U_{i n}}\left(\frac{1}{c-U_{i n}}+\frac{U_{i n}}{a_{i n}^{2}}\right) \int_{-1}^{1} \cos \left(\frac{n \pi}{2} y\right)^{2} d y \\
& =\frac{|A|^{2}}{\rho_{\text {in }}} \frac{c}{c-U_{i n}}\left(\frac{1}{c-U_{i n}}+\frac{U_{i n}}{a_{i n}^{2}}\right),
\end{aligned}
$$

a similar result being obtained for the anti-symmetric modes. The average net energy flux at the interface is given by,

$$
\begin{aligned}
\mathcal{J}_{j}(x) & =\left\langle J_{j}\left(x, 1^{-}, t\right)-J_{j}\left(x,-1^{+}, t\right)\right\rangle \\
& =\left(\Re\left(\hat{v}(1) \hat{p}^{+}(1)\right)-\Re\left(\hat{v}(-1) \hat{p}^{+}(-1)\right)\right) \rho_{i n} \frac{c}{c-U_{i n}} .
\end{aligned}
$$

For a duct mode, either $\hat{p}( \pm 1)=0$ (soft duct) or $\hat{v}( \pm 1)=0$ (hard duct), both of which lead to zero interface flux and thus to constant flux in the inner region. A first-order correction for the interface flux of modes departing from these behaviours is obtained by expanding the components $p^{\prime}$ and $v^{\prime}$ up to first order,

$$
\hat{p}=\hat{p}_{0}+\delta \hat{p}, \quad \hat{v}=\hat{v}_{0}+\delta \hat{v},
$$

the constraint at the interface being,

$$
\hat{p}_{0}+\delta \hat{p}=\left(\hat{v}_{0}+\delta \hat{v}\right) \mathcal{Z}_{V S} .
$$

For large $\mathcal{Z}_{V S}$ and $\hat{v}_{0}=0$, the leading terms are,

$$
\delta \hat{p}=0, \quad \delta \hat{v}=\frac{\hat{p}_{0}}{\mathcal{Z}_{V S}},
$$


while for small $\mathcal{Z}_{V S}$ and $\hat{p}_{0}=0$, we have,

$$
\delta \hat{p}=\hat{v}_{0} \mathcal{Z}_{V S}, \quad \delta \hat{v}=0 .
$$

Expressions for the interface energy flux under hard- and soft-walled duct approximations are obtained, respectively, by inserting 4.14 or 4.15 into 4.13 and 4.11 ,

$$
\begin{aligned}
& \mathcal{J}_{j}^{h}=2|A|^{2} \frac{c}{c-U_{\text {in }}} \Re\left(\mathcal{Z}_{V S}^{-1}\right), \\
& \mathcal{J}_{j}^{s}=2|A|^{2} \frac{\left|\xi_{\text {in }}\right|^{2}}{\rho_{\text {in }}^{2}\left|\alpha\left(c-U_{\text {in }}\right)\right|^{2}} \frac{c}{c-U_{\text {in }}} \Re\left(\mathcal{Z}_{V S}\right) .
\end{aligned}
$$

If $\left|\mathcal{J}_{j}^{s, h}\right| \ll|\mathcal{F}|$, a slowly varying amplitude, $A=A(x)$, may be assumed. The $x$-dependence of the amplitude is obtained by inserting equations 4.10 and 4.16 or 4.17 into 4.7 , giving,

$$
\frac{\partial|A(x)|^{2}}{\partial x}=-\frac{\mathcal{J}^{s, h}}{\mathcal{F}}|A(x)|^{2},
$$

and thus

$$
\left|A_{p}(x)\right|=\left|A_{p}(0)\right| \mathrm{e}^{-\frac{\mathcal{J}^{s}, h}{\mathcal{F}} x} .
$$

This spatial dependence can be seen as a wavenumber correction,

$$
\Delta \alpha_{i}=\frac{\mathcal{J}^{s, h}}{2 \mathcal{F}},
$$

which scales with $\Re\left(1 / \mathcal{Z}_{V S}\right)$ and $\Re\left(\mathcal{Z}_{V S}\right)$ for soft- and hard-walled duct modes, respectively. Using equation 3.3 the correction for a hard-walled duct becomes,

$$
\Delta \alpha_{i}=\frac{\mathcal{J}^{h}}{2 \mathcal{F}}=\frac{a_{\text {in }}^{2} \rho_{\text {in }}}{v_{\text {group }}} \Re\left(\mathcal{Z}_{V S}^{-1}\right) .
$$

Convective stability/instability is thus associated with negative/positive values of $\Re\left(\mathcal{Z}_{V S}\right)$. An analogous result is found for soft-walled ducts. A similar relation between stability in ducts and the real part of the impedance has been described by Rienstra \& Hirschberg (2018).

\subsection{Finite impedances in a compressible wake}

We now explore a first-order approximation for finite impedance (equation 4.20) in a Mach 0.9 wake. In order to isolate impedance effects, root loci are mapped as a function of $\rho_{r}$ : with $\omega$ and $T_{r}$ maintained constant, the propagative/evanescent characteristics of the soft-walled duct modes remain unaltered. The spatial eigenspectrum for $\rho_{r}=1$ is shown in figure 13. Modes in the left and right half-planes are, respectively, upstreamand downstream-travelling. All subsonic modes are constrained to the real $\alpha$ axis, and closely match the duct modes. Supersonic modes are all damped (i.e. they decay in the direction of generalised group velocity (Bers 1975)), and resemble attenuated duct modes.

Figure 14 maps loci of the modes indicated by solid markers in figure 13 as $\rho_{r}$ is varied. Supersonic modes make an excursion into the complex $\alpha$ plane, while subsonic modes are restricted to the real axis due to total internal reflection, as predicted by equation 4.2. Figure 15 compares $\alpha_{i}$ with the predictions of equation 4.20 , which accurately captures the spatial amplitude variation of these modes for most impedance values, failing only when the DVS transitions between soft- and hard-walled duct behaviours. The results illustrates how energy fluxes through the vortex-sheets constitute the stabilisation mechanism of the acoustic modes. 


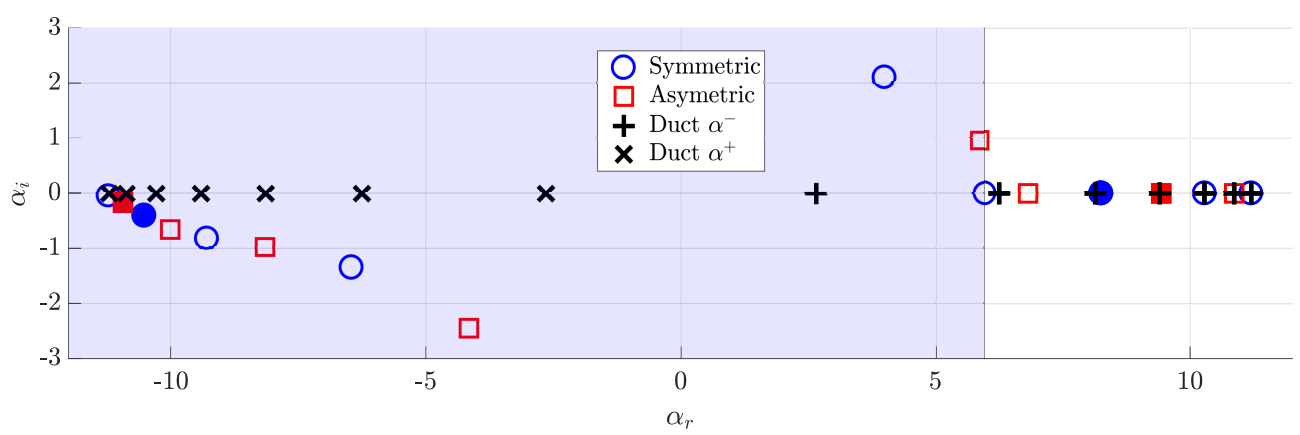

Figure 13: Roots of Equation 2.11 for $V_{\text {ref }}=0, M=0.9, \rho_{\text {in }, \text { out }}=1$, and $\omega=4 \pi$. Blue and red markers indicate symmetric and asymmetric modes, respectively, solid circles indicate the modes considered in figures 14 and 15. Hard- and soft-walled duct roots are indicated by " $\mathrm{x}$ " and "+", respectively. The blue region indicates supersonic phasevelocity with respect to the outer flow.

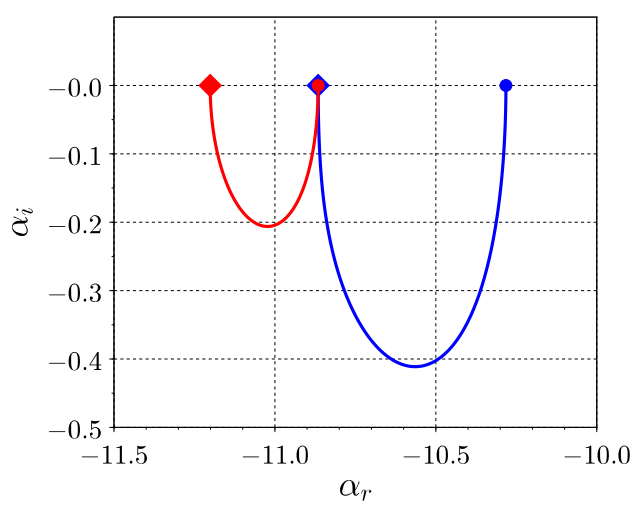

(a)

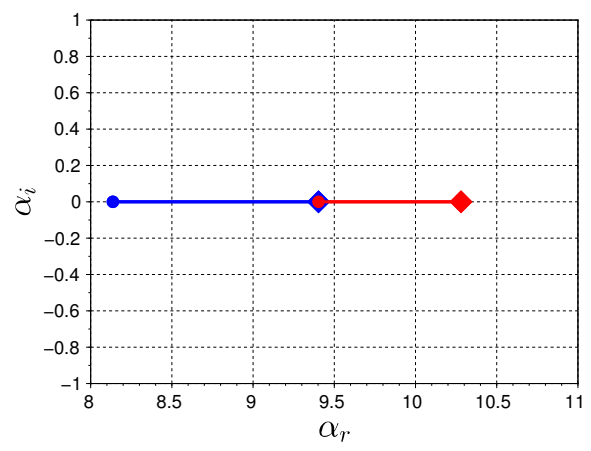

(b)

Figure 14: Loci, as a function of $\rho_{r}$, of upstream- (left) and downstream- (right) travelling modes, indicated in the left and right half planes of figure 13. Upstream-travelling modes move in to the complex $\alpha$ plane, while downstream-travelling modes remain on the real axis due to total reflection. Limits of hard- $\left(\rho_{r}=\infty\right)$ and soft-duct $\left(\rho_{r}=0\right)$ behaviour are indicated, respectively, by diamonds and circles. Red and blue lines represent antisymmetric and symmetric modes, respectively.

\subsection{Finite impedances in compressible jets}

We now consider a regime in which the real part of the impedance becomes negative. Inspection of equation 3.7 shows that the term $\rho_{\text {out }}\left(c-U_{\text {out }}\right)^{2}$ is always positive and real, $\mathrm{i} \xi_{\text {out }}$ is imaginary or positive real, and so only the term $\alpha /\left(c-U_{\text {in }}\right)$ may be either positive or negative. Expanding this term with equation 3.2, we obtain,

$$
\frac{\alpha}{c-U_{i n}}=\frac{\alpha^{2}\left(U_{i n}^{2} / a^{2}-1\right)}{\omega\left(-1 \pm U_{i n} / a \sqrt{1+\frac{(n \pi / 2)^{2}\left(U_{i n}^{2}-a^{2}\right)}{\omega^{2}}}\right)},
$$




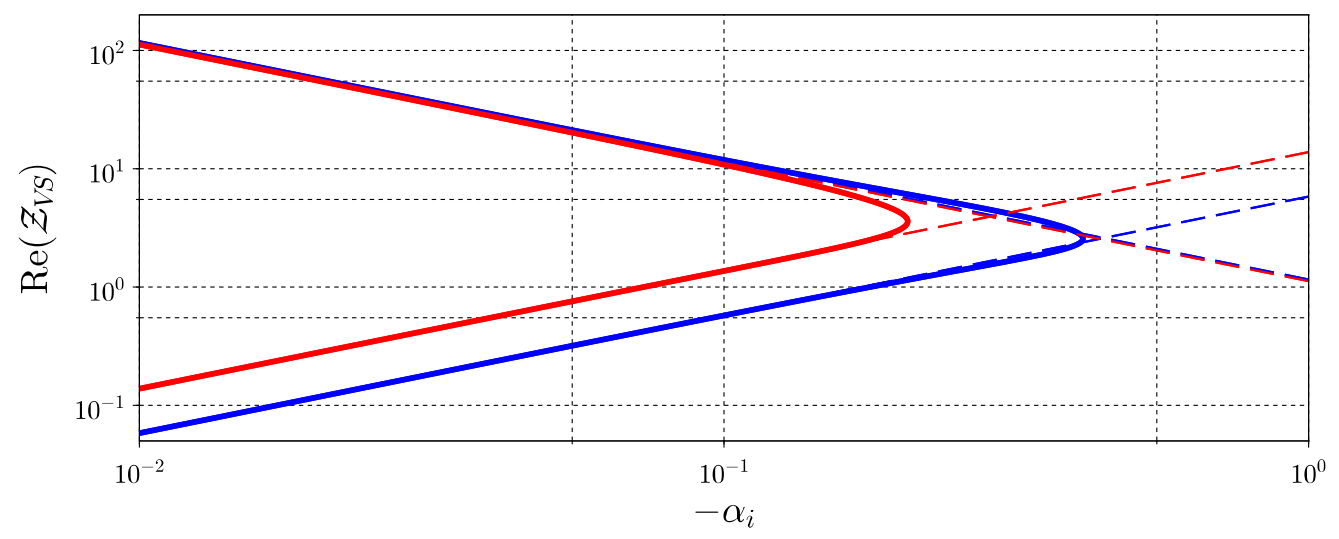

Figure 15: Relation between real part of impedance and $\alpha_{i}$ for the upstream-travelling modes of figure 13. Solid lines indicate dispersion-relation solutions, equation 2.11, and dashed lines show the first-order correction provided by equation 4.20.

where \pm corresponds to $\alpha^{+}$and $\alpha^{-}$modes. For a left-pointing supersonic jet $\left(U_{\text {in }}<-a\right)$, the ratio $\alpha /\left(c-U_{i n}\right)$ is always positive for $\alpha^{-}$modes and negative for $\alpha^{+}$. If they have supersonic phase speeds, the $\alpha^{-}$and $\alpha^{+}$modes become, respectively, stable and unstable, the number of supersonic modes increasing with Mach number.

Figure 16 shows the impedances associated with $\alpha^{+}$duct-mode Ansätze for a jet, as a function of Mach number. The impedance presents spikes whose locations collapse when scaled using duct-mode phase-speeds. The impedance spikes are related to transition between sub- and super-sonic phase-speeds: at the sonic limit, $\xi_{\text {out }} \rightarrow 0$, and thus, $\mathcal{Z}_{V S} \rightarrow$ $\infty$. The flow transitions from soft- to hard-walled duct behaviour.

Figure 17 compares the DVS modes of a supersonic jet to $\alpha^{-}$and $\alpha^{+}$duct modes, illustrating a correspondence between jet and duct behaviour. As predicted, waves corresponding to $\alpha^{-}$(respectively $\alpha^{+}$) duct modes exhibit spatial decay (respectively growth). The transition from soft- to hard-walled behavior is seen in 18. Supersonic $\alpha^{+}$ modes correspond to the unstable supersonic modes of Tam \& Hu (1989b): figure 17 corresponds to the transition between the subsonic and supersonic scenarios discussed in their work (cf. Figures 11 and 10, respectively, in Tam \& Hu (1989b)) . We thus see that the instability mechanism identified by Tam \& Hu (1989b) is underpinned by interaction of acoustic modes with the vortex sheet, and an associated influx of energy if the conditions discussed above are satisfied.

\subsection{Summary}

In this section we have shown how vortex-sheet impedance is a key parameter for the dynamics of acoustic modes. Analysis of the acoustic energy budget shows how outflux (respectively influx) of acoustic energy associated with a real, positive (respectively negative) vortex-sheet impedance is the key to stabilisation (respectively destablisation). We see how this can explain the stabilisation of acoustic modes in a compressible wake, and, perhaps more importantly, the appearance of unstable modes in supersonic jets (Tam \& Hu 1989b).

In the next section impulse responses are computed. The goal is two-fold: by constructing DVS impulse response we confirm the statement made in $\S 3.3$ regarding the stabilisation of jets, but not of wakes, that occurs on account of compressibility effects. And by constructing wave-packets for finite-thickness shear layers we show that the 

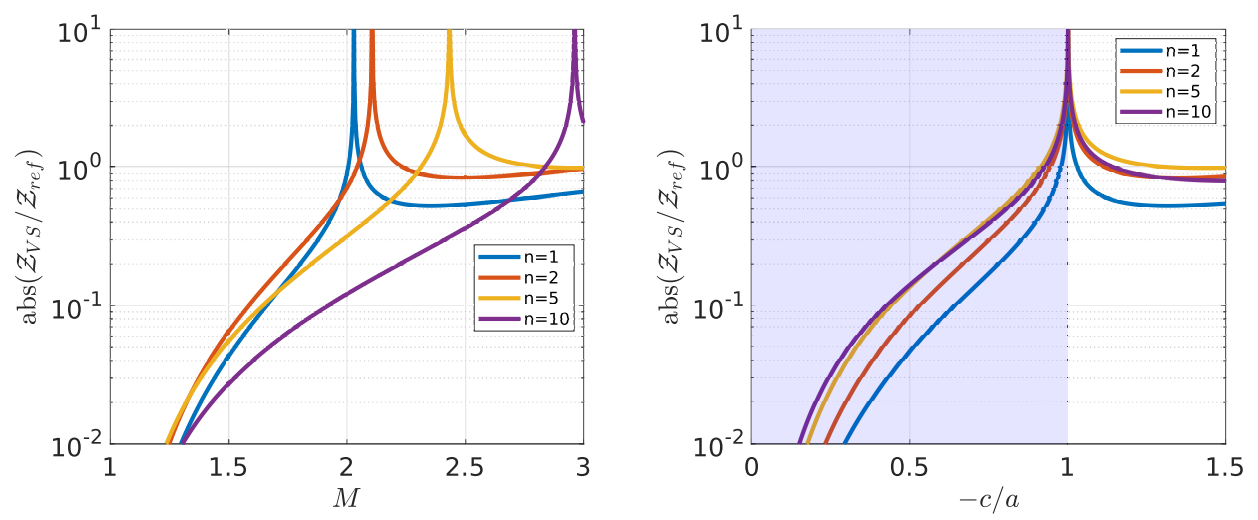

Figure 16: Absolute values of impedance for $\alpha^{+}$duct modes as a function of Mach number (left) and of duct-mode phase speed (right). Impedance peaks, found at different $M$ for each harmonic, occur when the phase speed of the mode is sonic. Results are for $\omega=\pi$, with the blue region indicating subsonic phase-speeds.

transition to absolute instability associated with saddles formed by $\mathrm{KH}$ and upstreamtravelling acoustic modes, presented in $§ 3.2$, also exists when finite-thickness shear layers are considered.

\section{Impulse Response}

\subsection{Impulse response of DVS}

The long-time impulse response is dominated by the dispersion relation dominant saddle point (Huerre \& Monkewitz 1985). The saddle points are identified by a null generalized group velocity

$$
\frac{\partial \omega}{\partial \alpha}=-\frac{\partial D_{d v s}}{\partial \alpha} / \frac{\partial D_{d v s}}{\partial \omega}=0,
$$

with $D_{d v s}$ given by equations 2.11 . The ill-posed nature of the vortex-sheet model has been evoked in $\S 3.2$ : vortex sheets possess unbounded growth rates with increasing $\omega$; the inclusion of a small shear-layer thickness regularises the problem. The absolute stability analysis thus amounts to a search for modes scaling with jet or wake width in the limit of small shear-layer thickness, as in Yu \& Monkewitz (1990). Because of this, we choose to refer to the DVS wavepacket (impulse response) as a pseudo wavepacket, calculated by mapping the dispersion-relation saddle points for each $V_{\text {ref }}$. The wave-packets so obtained can be used to infer the impulse response of any of the DVS base flows by a simple change of reference frame: for any $V_{\text {ref }}$ the maximum growth rate for a spacetime ray, $x / t=v^{*}$, is obtained from the saddle point with maximum $\omega_{0, i}$, of a flow with $V_{r e f}^{*}=V_{r e f}+v^{*}$. Details of the computation are provided in $\S$ B.1.

The pseudo wavepackets, whose growth rates are shown in figure 19, present two distinct regimes: the jet-like regime $\left(V_{\text {ref }}>0.5\right)$ is dominated by symmetric modes, and compressibility has a strong stabilising effect, while the wake-like regime $\left(V_{\text {ref }}<0.5\right)$ is dominated by antisymmetric modes with small sensitivity to compressibility. The large stabilisation effect in the jet regime is attributed to the presence of total internal reflection, as shown in figure 10 . 


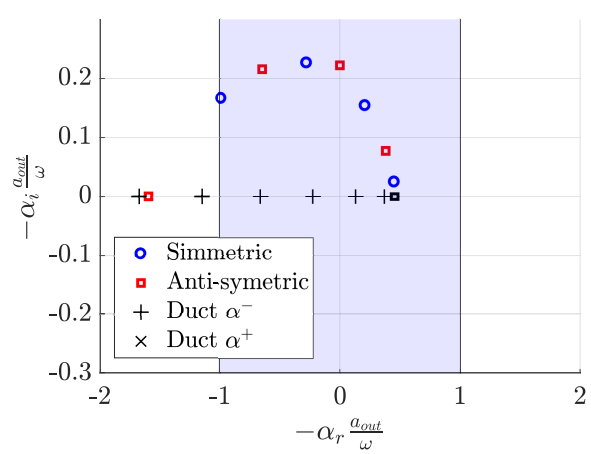

(a) $\mathrm{M}=1.2$

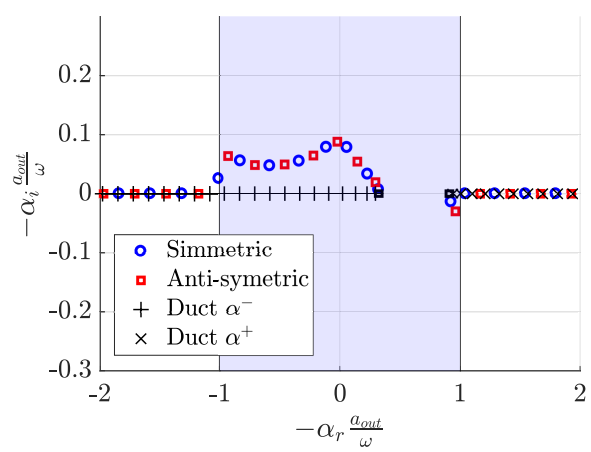

(c) $\mathrm{M}=2.1$

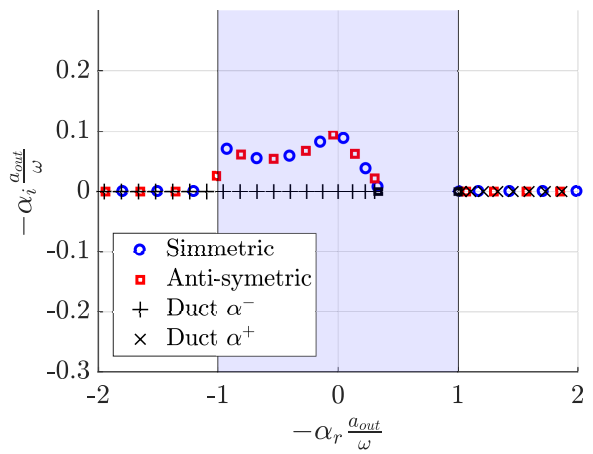

(b) $\mathrm{M}=2.0$

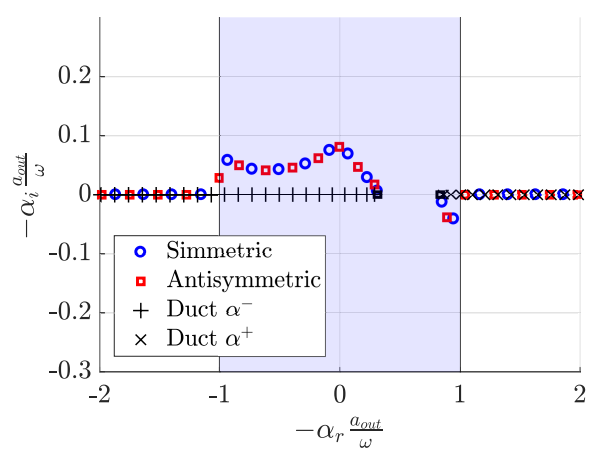

(d) $\mathrm{M}=2.2$

Figure 17: Instability of supersonic $\alpha^{+}$and stability of supersonic $\alpha^{-}$modes for a left pointing jet. Results are plotted in terms of $-\alpha$. All subsonic modes are neutrally stable, all supersonic modes being either stable $\left(-\alpha_{i}>0\right)$ or unstable $\left(-\alpha_{i}<0\right)$. Coloured markers indicate DVS modes, black markers indicated duct modes, with the $n=0$ mode identified by a square marker: this mode is not present in the soft-walled limit, appearing only at the transition to hard-walled behaviour. The blue region indicates supersonic phase speeds with respect to the outer region. Mach numbers 1.2, 2.0, 2.1, 2.2 from left to right, top to bottom. The plot is for constant Helmholtz numbers, $\omega=\pi$, and $\rho_{r}=T_{r}=1$.

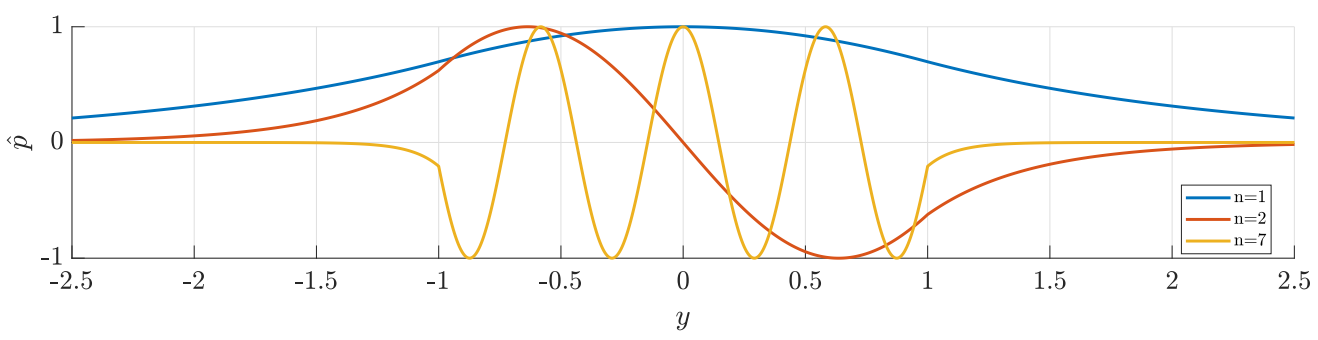

Figure 18: Pressure support for the first, second and seventh $\alpha^{+}$duct-like modes in a $M=2.0$ jet. Lower harmonics, which have near-sonic phase velocities with respect to the outer flow, resemble hard-walled duct modes; a transition to soft-duct behaviour is observed as the harmonic number increases. Results are for $V_{\text {ref }}=1$ and $\omega=\pi$. 


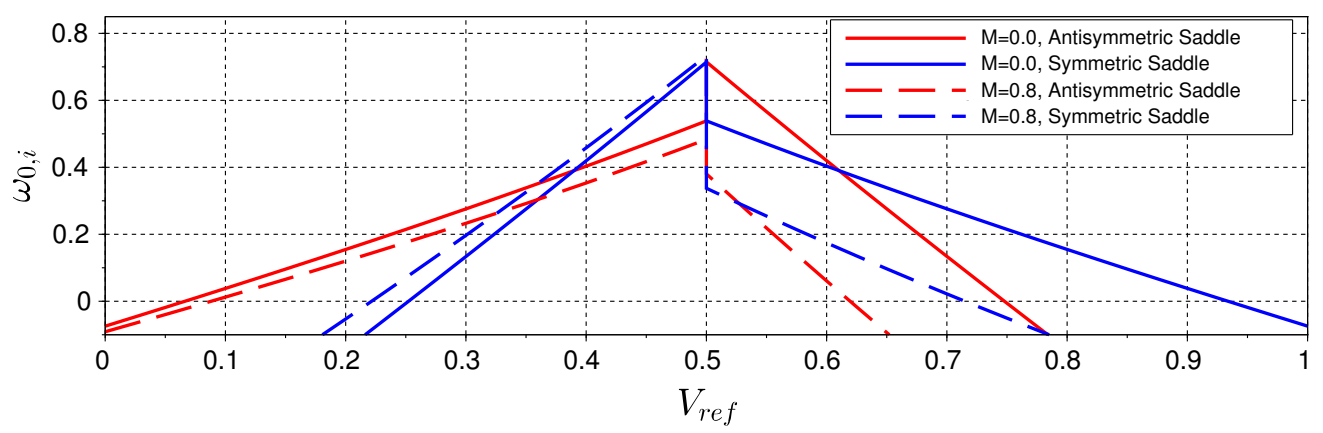

Figure 19: Growth rates of pseudo wave-packets of the DVS flow, for $T_{r}=1$.

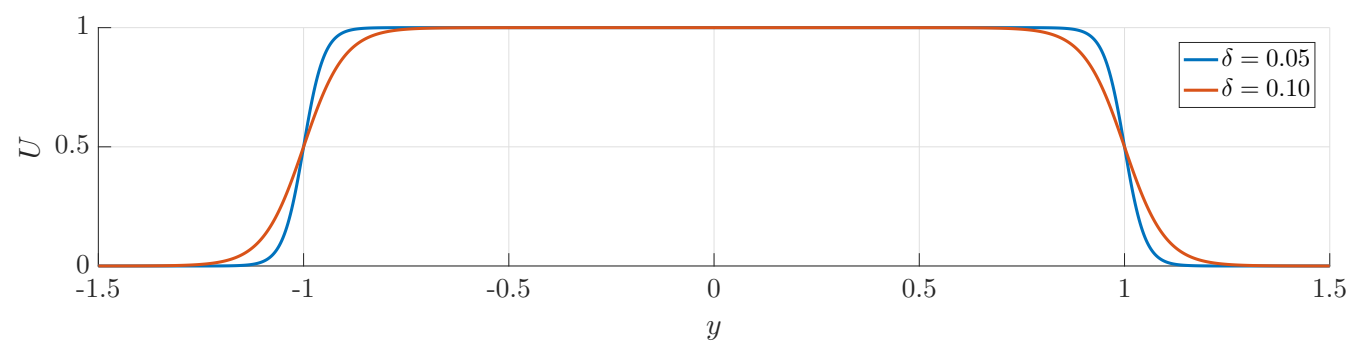

Figure 20: Illustration of a finite shear-layer jet obtained with Equation 5.2.

\subsection{Impulse response of finite shear-layer flows}

We extend the foregoing results to base flows with finite-thickness shear layers. We consider a profile modelled as,

$$
U(y)=\left(U_{\text {in }}-U_{\text {out }}\right)\left(\frac{\tanh \left(\frac{y+1}{\delta}\right)+\tanh \left(-\frac{y-1}{\delta}\right)}{2 \tanh \left(\frac{1}{\delta}\right)}\right)+U_{\text {out }},
$$

where $\delta$ controls the shear-layer thickness, as illustrated in Figure 20. The DVS model is recovered when $\delta \rightarrow 0$. We restrict our study to the incompressible regime. The modes were obtained by a discretisation of the Rayleigh equation using Gauss-Lobatto points. A pseudo-spectral code based on Chebyshev polynomials was used to obtain the derivatives at each point and standard generalised eigenvalue routines were used, as in Ormonde et al. (2018). Details of the procedures used can be found in Appendix B.2.

Figure 21 shows wavepacket growth rates for different values of $\delta$. For jets and wakes with small backflow, the instability scales with the distance between the vortex-sheets, corresponding to the DVS saddle points studied in the previous sections, slightly altered by shear-layer thickness. We refer to this saddle point as the column saddle, following Lesshafft \& Huerre (2007); it corresponds to mode II in Jendoubi \& Strykowski (1994). For large reverse flows $\left(0.2<V_{\text {ref }}<0.8\right)$ the instability scales with $\delta^{-1}$ and we refer to it as the shear-layer saddle, again following Lesshafft \& Huerre (2007); it corresponding to mode I of Jendoubi \& Strykowski (1994). This saddle point is characterised by large values of $\left|\alpha_{r}\right|$, suggesting negligible interaction between the vortex-sheets; it thus corresponds to the saddle point studied by Huerre \& Monkewitz (1985).

Although the shear-layer saddle point dominates most of the wavepacket, it is the column saddles that, due to backflow, first exhibit temporal growth, showing how acoustic modes are associated with the primary destabilisation mechanism of wakes and jets with 

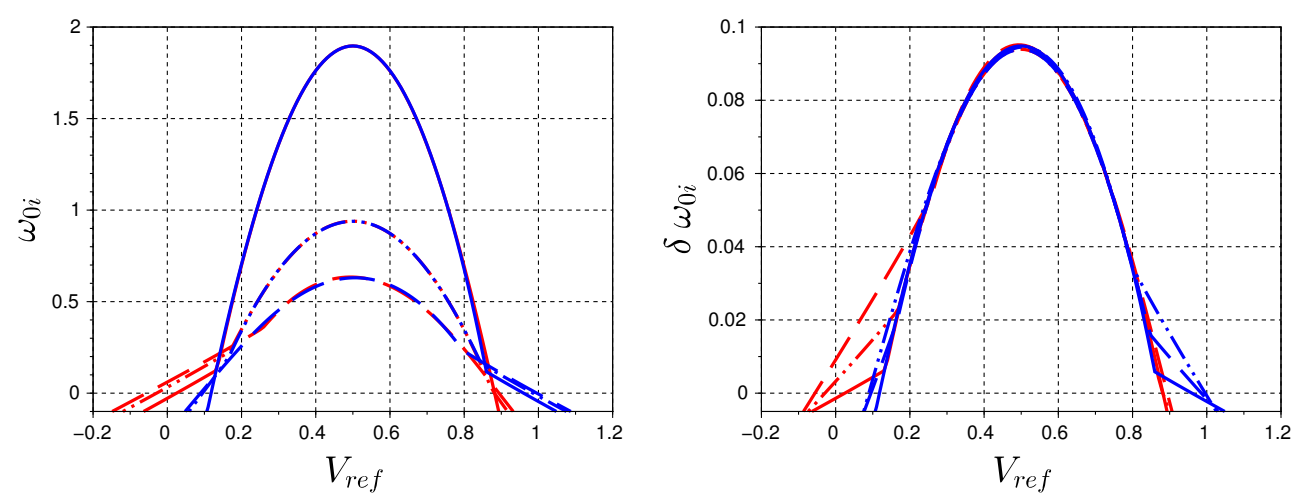

Figure 21: Wave-packets growth rates for $\delta=0.05,0.10$ and 0.20 (solid, dashed and dotted lines, respectively). Blue and red lines correspond to symmetric and antisymmetric modes, respectively. (a) Growth rates based on shear-layer separation; (b) Growth rates rescaled using the shear-layer thickness $\delta$.

finite-thickness shear layers. We also see that wakes are more sensitive to shear-layer thickness than jets, becoming unstable even in the absence of reverse flow due to finitethickness shear layers, while jets remain marginally stable.

A more complete investigation of the effects of shear layer thickness effects on compressible jets can be found in Tam \& Hu (1989a), where it is shown that subsonic modes may become unstable due to finite shear layer thickness; and in Parras \& Le Dizès (2010), where asymptotic expansions show that the presence of critical points (where $c=U(y)$ ) are related to the instability of the acoustic modes.

\section{Conclusion}

Modes of an acoustic nature have been identified in both compressible and incompressible double vortex-sheet (DVS) flow models, which we have configured in order to describe the behaviour of both jets and wakes. An analogy between these modes and acoustic modes found in ducts has been formulated, and it requires one of two $a$ priori conditions for its validity: (1) limiting values of vortex-sheet impedance, which are required to be either much larger or much smaller than a reference value; or, (2) the presence of total reflection, with evanescent wave dynamics in the outer flow. Duct-like modes are found in both jets and wakes over an extensive parameter space, generalising the analysis of Towne et al. (2017) for a broader class of flows; and, in particular, in the incompressible limit, where acoustic waves become evanescent pressure modes.

We show how upstream-travelling acoustic modes are central for a complete understanding of absolute instability. Specifically, deterioration of their duct-like behaviour appears as a necessary condition for the onset of instability. When the acoustic modes are duct-like, jets and wakes behave as waveguides, and upstream-travelling modes have negligible interaction with Kelvin-Helmholtz modes. A departure from duct-like behaviour enables interaction between acoustic and $\mathrm{KH}$ modes, a neutral saddle point eventually forming between the two and marking the onset of absolute instability. This scenario was verified to hold in both incompressible and compressible regimes.

The mechanisms we discuss are similar in some respects to those discussed by Healey (2009), where confinement effects were shown to lead to absolute instability due to the action of acoustic modes. Healey's acoustic modes scale with the distance between 
confining walls, which are imposed. In our work they scale with the distance between vortex-sheets, and the "wall" behaviour is an intrinsic part of the flow dynamics.

The phenomena we consider are also relevant for global instabilities, as for slowlydiverging base flows, global modes may be built using the WKB method (Monkewitz et al. 1993; Siconolfi et al. 2017), which shows the global instability to arise on account of local pockets of absolute instability. Investigating of these modes in non-parallel systems would thus appear worthwhile, as the global stability of a given system might be modified/controlled via an appropriate manipulation of the acoustic mode.

The departure from duct-like behaviour was explored via an energy balance analysis, derived in $\S 4$. Energy fluxes through the vortex-sheets are associated with spatial growth or decay of the acoustic-mode amplitude. Energy outflow and inflow were shown to underpin, respectively, the stabilisation and destabilisation of acoustic modes, and it was shown how this behaviour is associated with the unstable supersonic modes identified by Tam \& Hu (1989b). First order corrections were derived in order to described acousticmode amplitude variations close to the duct-limit conditions.

Impulse responses of jets and wakes with vanishing and finite-thickness shear layers were computed, supporting previous results and showing that the main conclusions of our analysis also hold for flows with finite-thickness shear layers, and are not an artefact of the vortex-sheet model. The saddle points associated with finite-thickness shear-layer flows are most relevant for absolute instability when strong reverse flow exists. For jets and wakes with little or no reverse flow, the absolute/convective stability behaviour is found to be dominated by interaction between Kelvin-Helmholtz and the upstream-travelling, acoustic modes we consider.

The analysis opens up new possibilities for the study of global instability in free-shear flows. Clarification of the role played by acoustic modes has permitted a unified explanation of known, but previous unconnected, trends found in the literature: (1) transition from convective to absolute instability due to backflow, wake cooling (Monkewitz 1988) and jet heating (Monkewitz \& Sohn 1988); (2) the differing symmetries of unstable wakes and jets (Yu \& Monkewitz 1990); (3) acoustic wave trapping (Schmidt et al. 2017; Towne et al. 2017); and, (4) the unstable supersonic jet modes of Tam \& Hu (1989b).

Perhaps more interestingly, the fact that acoustic phenomena are central in the destabilisation of wakes (leading to the well-known von Kármán vortex street in the saturated limit) and hot jets, suggests the possibility of novel approaches for flowcontrol. Instead of targeting the classical Kelvin-Helmholtz instability, one may consider modifying the behaviour of a flow by changing its acoustic properties.

\section{Acknowledgements}

A. V. G. Cavalieri acknowledges financial support by $\mathrm{CNPq}$ (grant number 310523/2017-6). E. Martini acknowledges financial support from CAPES (grant number $88881.190271 / 2018-01)$.

\section{Appendix A. Review of duct acoustics}

Given a duct containing a uniform, two-dimensional flow of velocity $U$, the linearised Euler equations can be simplified, leading to the convected wave equation,

$$
\left(\frac{\partial}{\partial t}+U \frac{\partial}{\partial x}\right)^{2} p^{\prime}-a^{2}\left(\frac{\partial^{2}}{\partial x^{2}}+\frac{\partial^{2}}{\partial y^{2}}\right) p^{\prime}=0
$$


where $t$ denotes time, $(x, y)$ streamwise and wall-normal Cartesian coordinates, $p^{\prime}$ pressure fluctuations and $a$ the speed of sound. We assume hard walls at $y= \pm 1$, and associated boundary conditions $\partial p^{\prime} / \partial y( \pm 1)=0$.

Using the Ansatz

$$
p^{\prime}(x, y, t)=\mathrm{e}^{i(\alpha x-\omega t)}(A \sin (\beta y)+B \cos (\beta y)),
$$

where $\alpha$ and $\beta$ are streamwise, $x$ and wall-normal, $y$, wavenumbers, and $\omega$ is frequency, the dispersion relation,

$$
(\omega-U \alpha)^{2}=a^{2}\left(\alpha^{2}+\beta^{2}\right)
$$

is obtained. Boundary conditions enforce $\beta=\pi n / 2$, where $n$ is an integer harmonic number. Odd values of $n$ imply $B=0$, leading to antisymmetric pressure distributions, while even values imply $A=0$, and symmetric pressure distributions. Solving for the horizontal phase speed, $c=\omega / \alpha$, as a function of $\alpha$ and $\beta$, leads to modes corresponding to a temporal stability analysis, given by,

$$
c=U \pm a \sqrt{1+\frac{\beta^{2}}{\alpha^{2}}}
$$

and with group velocity,

$$
v_{\text {group }}=\frac{\partial \omega}{\partial \alpha}=U+\frac{a}{(c-U)} a .
$$

For $U=0$, the roots of equation A 4 correspond to waves travelling to the right (plus sign) and to the left (minus sign). For non-zero $U$ the modes are convected by the mean flow, as illustrated in figures 22 (a) and (b), where the phase velocity for a given $\alpha$ and $\beta$ is shown. We note that for $U=0$, modes have phase and group velocities of the same sign, whereas for $U=0.9$ in some cases we have a mode with negative phase-speed and positive group velocity. Solving for $\alpha$, assuming fixed $\omega$ and $\beta$,

$$
\alpha=\frac{-U \omega \pm a \sqrt{\omega^{2}-(n \pi / 2)^{2}\left(a^{2}-U^{2}\right)}}{a^{2}-U^{2}},
$$

giving modes that would be consistent with a spatial stability analysis.

Different scenarios emerge for real or complex $\alpha$. If $\alpha$ is a purely real number it represents propagative modes, whose amplitudes are constant along the duct. They are found for,

$$
\omega^{2}>(n \pi / 2)^{2}\left(a^{2}-U^{2}\right) .
$$

At lower frequencies, $\alpha$ has a non-zero imaginary part and the modes are evanescent, presenting exponential spatial decay in amplitude. For $U<a$, the threshold between evanescent and propagative modes is given by the cut-on frequency,

$$
\omega_{\text {cut on }}=n \frac{\pi}{2} \sqrt{a^{2}-U^{2}},
$$

while for $U>a$ (supersonic flows) all acoustic modes are propagative and downstreamtravelling. Only evanescent modes are present in the incompressible limit $(a \rightarrow \infty)$.

Figure 22 (c) and (d) show phase and group velocities for a fixed $\beta=\pi / 2$ (first harmonic), showing that in the presence of a mean flow and for frequencies slightly above the cut-on value, modes with both signs can have subsonic phase speeds. These are modes that are evanescent for $U=0$ but become propagative when flow is present, or equivalently, when the observer's frame of reference changes (Rienstra \& Hirschberg 2018). 


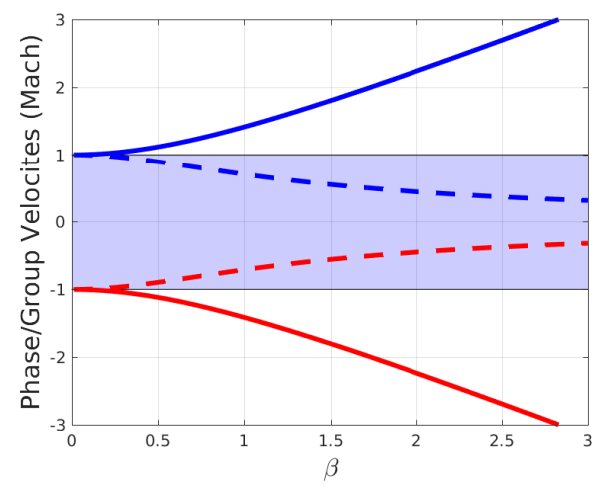

(a) $U=0.0$

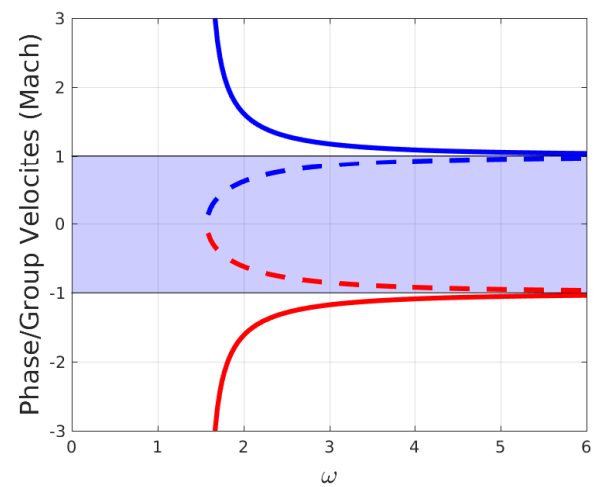

(c) $U=0.0$

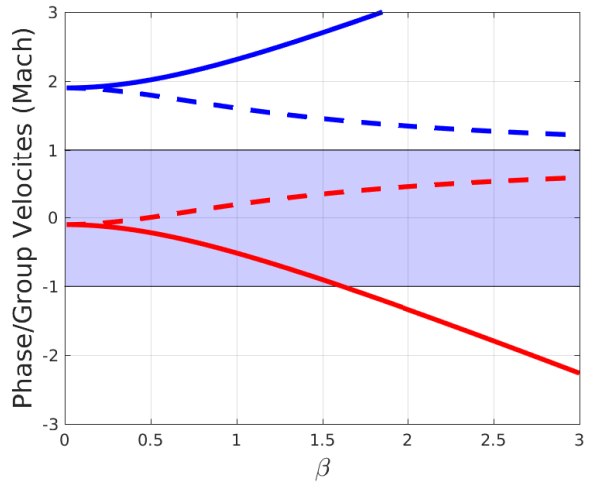

(b) $U=0.9$

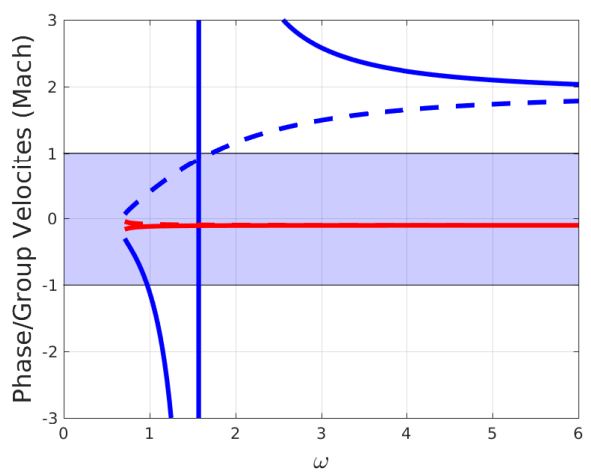

(d) $U=0.9$

Figure 22: Behavior of phase (solid) and group (dashed) velocities for the positive (red) and negative (blue) signs for propagative modes. Shaded regions indicates the subsonic velocities on the laboratory reference frame. For figures (a) and(b) $a=1, \omega=1$ and $\alpha(\omega, \beta)$, while for (c) and(d) $a=1$ and $\beta=\pi / 2$ were used, with $\omega(\alpha, \beta)$. The interdependency of $\alpha, \beta, \omega$ are given by equation A 3 . Note that the boundary conditions limit the allowable values of $\beta$ to multiples of $\pi / 2$, nevertheless we keep all values of $\beta$ for a clearer visualization of the trends.

Ducts with acoustically soft, or pressure-release, walls have as boundary conditions, $p( \pm 1)=0$. Modes in these ducts share the same properties and dispersion relation as the hard duct discussed above, differing in that the plane-wave mode $(n=0)$ only satisfies the boundary conditions for the hard-wall case, and in that modes have opposite symmetries in soft and hard ducts: odd modes $(n=1,3,5, \ldots)$ are antisymmetric and symmetric for hard and soft ducts, respectively, and the opposite being true for even modes $(n=2,4,6, \ldots)$. 


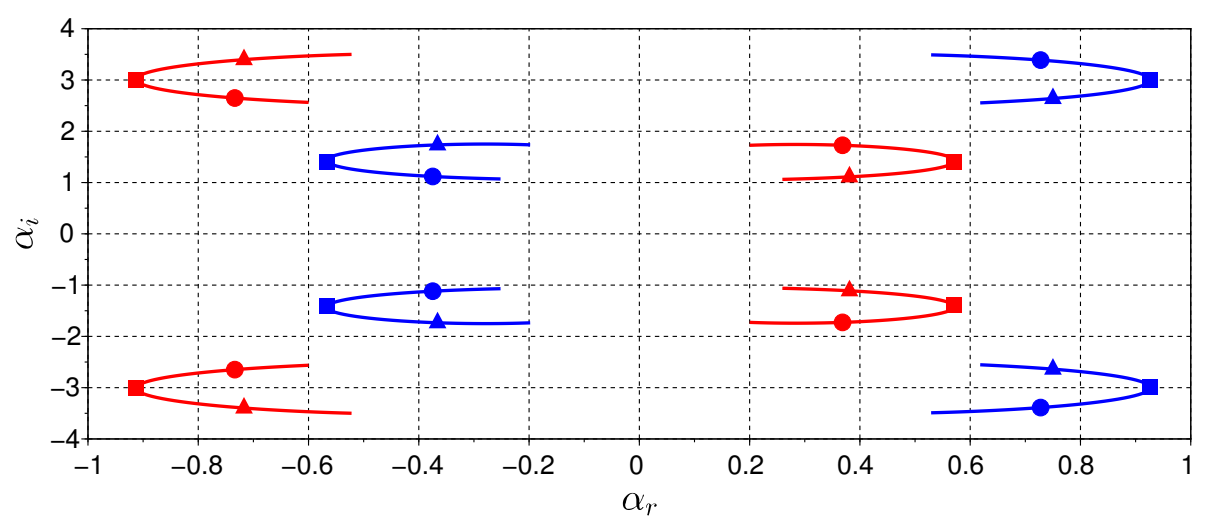

Figure 23: Locus of eight DVS saddles point with increasing $V_{\text {ref }}$. Symmetric and antisymmetric mode saddles in red and blue. The triangle, square and circle markers correspond to $V_{\text {ref }}=0,0.5$ and 1.0 .

\section{Appendix B. Impulse response}

\section{B.1. DVS pseudo wave-packets}

Approximate DVS saddles were found by tracing root loci with increasing $\omega_{r}$ for different values of $\omega_{i}$. Results were then refined by numerically solving

$$
D_{d v s}(\alpha, \omega)=0, \text { and } \quad \frac{\partial \omega}{\partial \alpha}=\frac{\partial D_{d v s}}{\partial \alpha} / \frac{\partial D_{d v s}}{\partial \omega}=0,
$$

where $D_{d v s}$ is the dispersion relation of the DVS. The saddles loci with $V_{\text {ref }}$ were obtained by making small increments on it and using previous results as initial guesses. As in Jendoubi \& Strykowski (1994), many of saddle points were found, each comprising the $\mathrm{KH}$ branch and an acoustic-mode branch. The locus of eight of these saddles with $V_{\text {ref }}$, defined in equation 2.2, are shown in figure 23. For all parameters studied the dominant saddle was formed by the first harmonic acoustic mode.

Saddles in the first and third quadrant were found to be formed by a pair of upstreamor a pair of downstream-traveling modes, and thus are not relevant in the impulse response calculation (Huerre \& Monkewitz 1990; Huerre et al. 2000). Saddles of the second and fourth quadrants are the relevant ones, showing a more complex behavior.

For $V_{\text {ref }}<0.5$ fourth quadrant saddles are formed by an upstream and a downstream mode. However when $V_{\text {ref }}=0.5$ the downstream mode becomes an upstream mode, thus the saddle is suddenly not "valid" anymore. This process is illustrated in figure 24 . A similar but opposite behavior is found in the second quadrant, for $V_{\text {ref }}<0.5$ the saddles are all formed by two upstream modes, with one upstream mode becoming a downstream mode at $V_{\text {ref }}=0.5$.

This phenomena is connected to KHs mode group velocity, which can be related to the mean of inner and outer flow speeds. Clearly, for $V_{\text {ref }}=0$, the KH mode travels towards positive $\mathrm{x}$ for the ideal wake, but for $V_{\text {ref }}=1$ the same mode travels towards negative $\mathrm{x}$ for the left-pointing jet. At $V_{\text {ref }}=0.5$ the $\mathrm{KH}$ transitions from a upstream- to an downstream-traveling mode, invalidating one saddle and validating the other. This leads to a discontinuities in growth rates of both symmetric and antisymmetric pseudo wavepacket, as seen in figure 19. This discontinuities are a consequence of the zero thickness shear layers, disappearing when finite shear layers are considered, as explored in section 5.2 . 


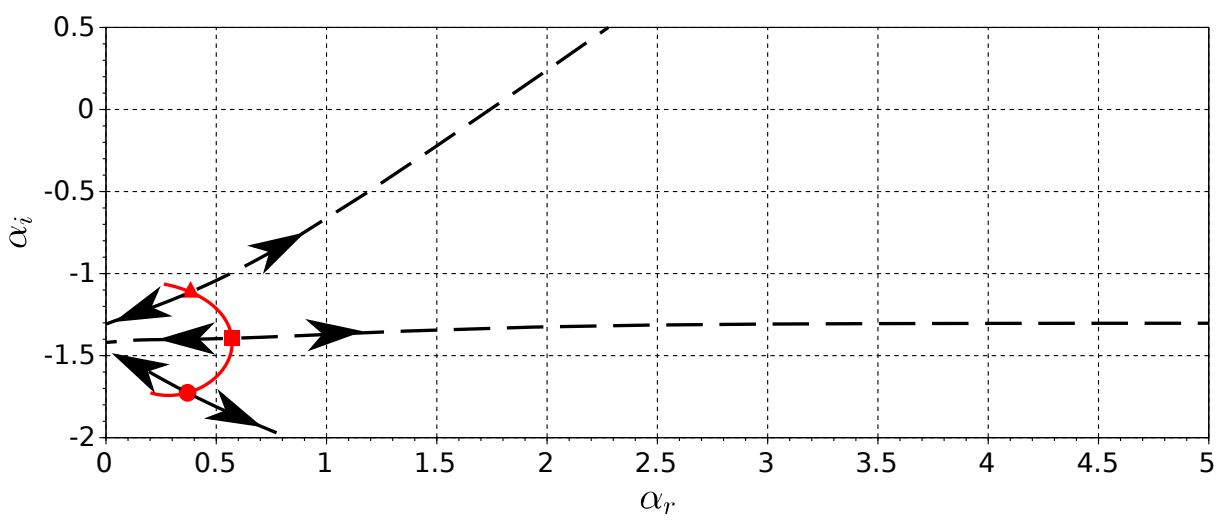

Figure 24: Briggs criteria for 4th quadrant saddle points for different $V_{\text {ref }}$. Red line represents the saddle point locus, black dashed lines show the path for both of the saddle roots with increasing $\omega_{i}$, with arrows indicating direction of increasing $\omega_{i}$.

\section{B.2. Finite shear-layer thickness wave-packet}

Finite shear-layer effects are studied with profiles given by

$$
U(y)=\left(U_{\text {in }}-U_{\text {out }}\right)\left(\frac{\tanh \left(\frac{y+1}{\delta}\right)+\tanh \left(-\frac{y-1}{\delta}\right)}{2 \tanh \left(\frac{1}{\delta}\right)}\right)+U_{\text {out }},
$$

where $\delta$ controls the thickness of the mixing layer. The DVS model is recovered by when $\delta \rightarrow 0$. Pseudo-spectral methods using Chebyshev polynomials and Gauss-Lobatto points in order to obtain the derivatives ot each point and standard generalized eigenvalue routines were used, as in Ormonde et al. (2018). Two consecutive mappings were used in order to improve vortex-sheet resolution,

$$
\begin{aligned}
f_{1}(y) & =L \frac{y}{\sqrt{1-y^{2}}} \\
f_{2}^{-1}(y) & =y+a_{2}\left(\tanh \left(\frac{y-1}{a_{1}}\right)+\tanh \left(\frac{y+1}{a_{1}}\right)\right),
\end{aligned}
$$

with $L=2, a_{1}=1.2 \delta, a_{2}=2$. The mapping given by $f_{1}$ corresponds to the transformation from Chebyshev to rational Chebyshev polynomials, and by $f_{2}$ concentrates collocation points around the vortex-sheets. The impulse response was obtained by looking for saddle points in the complex $\alpha$ plane. Different runs were made varying $\omega_{(r, i)}$ between $\omega_{g,(r, i)} \pm 0.05$, where $\omega_{g}$ is the approximate saddle location. Saddles wave number $\left(\alpha_{0}\right)$ and frequency $\left(\omega_{0}\right)$ were estimated by a second order fit of $\omega$ and $\alpha$ values of the roots closer to the estimated saddle position.

The locus of saddle points as a function of $V_{\text {ref }}$ for two shear-layer thicknesses, $\delta=0.05$ and 0.1 , is shown in figure 25. Two types of saddle can be observed. Column saddles occurs for low $|\alpha|$ on both thicknesses and scale with the distance between shear layers. Shear-layer saddles scales with shear-layer thickness, occurring for larger values of $|\alpha|$ for thin layers.

The shear-layer saddles corresponds to finite-thickness single vortex-sheet saddles, first studied by Huerre \& Monkewitz (1985): when $\delta \ll 1$, they occur for $|\Re(\alpha)|>>1$, and thus the wavelength is much smaller than the distance between shear layers, effectively decoupling their behavior and recovering single vortex-sheet results, which is reflected by 


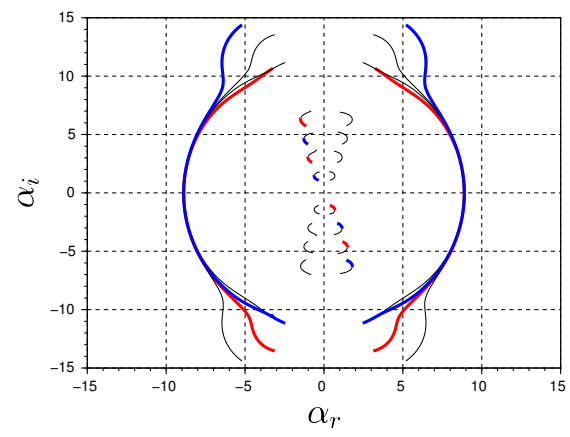

(a) $\delta=0.05$

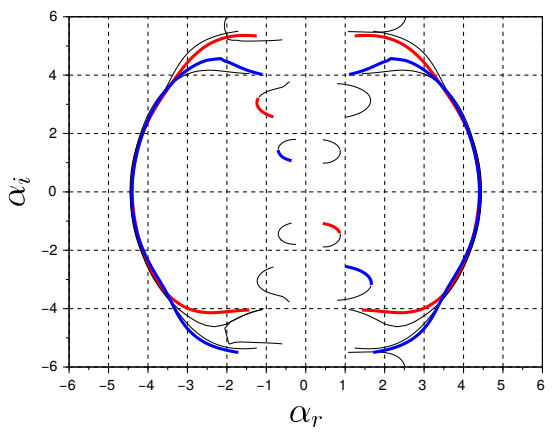

(b) $\delta=0.10$

Figure 25: Saddle locus in the complex $\alpha$-plane with $V_{\text {ref }}$. Blue and red lines indicate "valid" (saddle formed by upstream- and downstream-traveling modes) symmetric and antisymmetric modes. Black lines indicate invalid saddles. For small values of $\delta$ theres a clear distinction between columns and shear layer saddles.

similar growth rates of symmetric and antisymmetric asbolute instabilities. The range of $V_{\text {ref }}$ where the shear-layer saddle dominates has counterflow in the shear layer, and thus corresponds to the cases of absolute instability of shear-layers due to backflow. Lesshafft \& Huerre (2007) shear-layer saddles scales with round jets shear-layer thickness, equivalent to results seen in figure 21 for the DVS model.

\section{Appendix C. Free vibration of a string with discontinuous density}

A vibrating string obeys the following equation

$$
\frac{\partial^{2} f}{\partial t^{2}}(y, t)+\frac{T}{\rho(y)} \frac{\partial^{2} f}{\partial y^{2}}(y, t)=0,
$$

where $f$ is the string displacement and $T$ is the string tension, which is constant in the absence of external forcing. We consider here a model where

$$
\rho(x)= \begin{cases}\rho_{\text {out }} & ,|y|>1 \\ \rho_{\text {int }} & ,|y|<1\end{cases}
$$

We can solve the equation on each of the three domains $(y<1,|y|<1$ and $y>1)$ and then patch the solutions using as interface conditions the continuity of string displacement and force balance,

$$
\begin{aligned}
f\left(+1^{+}, t\right) & =f\left(+1^{-}, t\right) & \frac{T}{\rho_{\text {out }}} \frac{\partial f}{\partial y}\left(+1^{-}, t\right) & =\frac{T}{\rho_{\text {in }}} \frac{\partial f}{\partial y}\left(+1^{+}, t\right) \\
f\left(-1^{+}, t\right) & =f\left(-1^{-}, t\right) & \frac{T}{\rho_{\text {out }}} \frac{\partial f}{\partial y}\left(-1^{-}, t\right) & =\frac{T}{\rho_{\text {in }}} \frac{\partial f}{\partial y}\left(-1^{+}, t\right) .
\end{aligned}
$$

As boundary conditions we use that for $|y| \rightarrow \infty$ we retain only waves moving away from the origin. Assuming symmetric disturbances around $y=0$ we have as solutions in each domain 


$$
f(x, t)=\left\{\begin{array}{ll}
A \mathrm{e}^{-i \alpha_{\text {out }} y-i \omega t}, & y>1 \\
\cos \left(\alpha_{\text {in }} y\right) \mathrm{e}^{-i \omega t}, & 0<y<1
\end{array},\right.
$$

where $\alpha_{\text {in }}=\omega \sqrt{T / \rho_{\text {in }}}$ and $\alpha_{\text {out }}=\omega \sqrt{T / \rho_{\text {out }}}$. Using the interface conditions we arrive at the dispersion relation

$$
\begin{aligned}
\tan \left(\alpha_{\text {in }}\right)=i\left(\frac{\rho_{\text {out }}}{\rho_{\text {in }}}\right)^{3 / 2} \quad \alpha_{\text {in }} & =\tan ^{-1}\left(i\left(\frac{\rho_{\text {out }}}{\rho_{\text {in }}}\right)^{3 / 2}\right)+n \pi \\
\alpha_{\text {out }} & =\left(\tan ^{-1}\left(i\left(\frac{\rho_{\text {out }}}{\rho_{\text {in }}}\right)^{3 / 2}\right)+n \pi\right) \sqrt{\frac{\rho_{\text {in }}}{\rho_{\text {out }}}}
\end{aligned}
$$

A similar equation is found for the antisymmetric mode, but with a cotangent instead of the tangent function in equations $\mathrm{C} 6$ and $\mathrm{C} 7$.

The amplitude of the outgoing wave is

$$
A=\frac{\cos \left(\alpha_{\text {in }}\right)}{\mathrm{e}^{-i \alpha_{\text {out }}}} .
$$

In the limit when $\rho_{\text {in }} / \rho_{\text {out }} \rightarrow 0, \alpha_{\text {in }}$ tends to $(n+1 / 2) \pi$, and $A \rightarrow 0$, while when $\rho_{\text {in }} / \rho_{\text {out }} \rightarrow \infty,|A| \rightarrow 1$. Interesting aspects of these limits is the emulation of displacement constrains or of force relief constrains at $x= \pm 1$, as seen in figure 11 . For finite values of $\rho_{\text {in }} / \rho_{\text {out }}, \omega$ has a negative imaginary part, indicating a decay of amplitude. When the density ratio is large, the amplitude decay is small because the amplitude of the outgoing waves (A) is small, while on the limit of small density ratio even with unitary amplitude of outgoing waves, the energy of those waves is small when compared with the vibration energy of the inner string.

\section{REFERENCES}

Bers, Abraham 1975 Linear waves and instabilities. In Plasma physics-les houches 1972.

Bogey, Christophe \& Gojon, Romain 2017 Feedback loop and upwind-propagating waves in ideally expanded supersonic impinging round jets. Journal of Fluid Mechanics 823, $562-591$.

Brès, Guillaume A., Jordan, Peter, Jaunet, Vincent, Le Rallic, Maxime, Cavalieri, André V. G., Towne, Aaron, Lele, Sanjiva K., Colonius, Tim \& Schmidt, Oliver T. 2018 Importance of the nozzle-exit boundary-layer state in subsonic turbulent jets. Journal of Fluid Mechanics 851, 83-124.

Briggs, RICHARD J 1964 Electron-stream interaction with plasmas mit press. Monograph MIT, Cambridge USA (29).

Chomaz, Jean-Marc, Huerre, Patrick \& Redekopp, Larry G. 1991 A frequency selection criterion in spatially developing flows. Studies in Applied Mathematics 84 (2), 119-144.

Gojon, Romain \& Bogey, Christophe 2017 Flow structure oscillations and tone production in underexpanded impinging round jets. AIAA Journal 55 (6), 1792-1805.

Gojon, Romain, Bogey, Christophe \& Marsden, Olivier 2015 Large-eddy simulation of underexpanded round jets impinging on a flat plate 4 to 9 radii downstream from the nozzle. AIAA Paper 2210, 2015.

Goldstein, Marvin E 1976 Aeroacoustics. New York, McGraw-Hill International Book Co., 1976. $305 \mathrm{p}$. .

Healey, JJ 2009 Destabilizing effects of confinement on homogeneous mixing layers. Journal of Fluid Mechanics 623, 241-271.

Huerre, Patrick, Batchelor, GK, Moffatt, HK \& Worster, MG 2000 Open shear flow instabilities. Perspectives in fluid dynamics pp. 159-229. 
Huerre, Patrick \& Monkewitz, Peter A 1985 Absolute and convective instabilities in free shear layers. Journal of Fluid Mechanics 159, 151-168.

Huerre, Patrick \& Monkewitz, Peter A 1990 Local and global instabilities in spatially developing flows. Annual review of fluid mechanics 22 (1), 473-537.

Jendoubi, S \& Strykowski, PJ 1994 Absolute and convective instability of axisymmetric jets with external flow. Physics of Fluids 6 (9), 3000-3009.

Jordan, Peter, Jaunet, Vincent, Towne, Aaron, Cavalieri, André V. G., Colonius, Tim, Schmidt, Oliver \& Agarwal, Anurag 2018 Jet-flap interaction tones. Journal of Fluid Mechanics 853, 333-358.

Karamcheti, Krishnamurty 1956 Sound radiation from surface cutouts in high speed flow. $\mathrm{PhD}$ thesis, California Institute of Technology.

Krothapalli, A 1985 Discrete tones generated by an impinging underexpanded rectangular jet. AIAA journal 23 (12), 1910-1915.

Krothapalli, A, Rajkuperan, E, Alvi, F \& Lourenco, L 1999 Flow field and noise characteristics of a supersonic impinging jet. Journal of Fluid Mechanics 392, 155-181.

Lamb, Horace 1945 Hydrodynamics dover. New York 43.

Lessen, M, Fox, JA \& Zien, HM 1965 The instability of inviscid jets and wakes in compressible fluid. Journal of Fluid Mechanics 21 (1), 129-143.

Lesshafft, Lutz \& Huerre, Patrick 2007 Linear impulse response in hot round jets. Physics of Fluids 19 (2), 024102.

Monkewitz, Petera \& Sohn, Kinod 1988 Absolute instability in hot jets. AIAA journal 26 (8), 911-916.

Monkewitz, Peter A. 1988 The absolute and convective nature of instability in two dimensional wakes at low reynolds numbers. The Physics of Fluids 31 (5), 999-1006, arXiv: http://aip.scitation.org/doi/pdf/10.1063/1.866720.

Monkewitz, Peter A., Huerre, Patrick \& Chomaz, Jean-Marc 1993 Global linear stability analysis of weakly non-parallel shear flows. Journal of Fluid Mechanics 251, $1-20$.

Noack, Bernd R. \& Eckelmann, Helmut 1994 A global stability analysis of the steady and periodic cylinder wake. Journal of Fluid Mechanics 270, 297-330.

Ormonde, Pedro C, Cavalieri, André VG, da Silva, Roberto Ga \& Avelar, Ana C 2018 Passive control of coherent structures in a modified backwards-facing step flow. Experiments in Fluids $\mathbf{5 9}$ (5), 88.

Parras, Luis \& Le Dizès, StÉPhane 2010 Temporal instability modes of supersonic round jets. Journal of Fluid Mechanics 662, 173-196.

Pierce, A. 1981 Acoustics: An introduction to its physical principles and applications. NY Mc Graw-Hill .

Powell, Alan 1953 The noise of choked jets. The Journal of the Acoustical Society of America $25(3), 385-389$.

Rienstra, SW \& Hirschberg, A 2018 An introduction to acoustics .

Rossiter, JE 1964 Wind tunnel experiments on the flow over rectangular cavities at subsonic and transonic speeds. Tech. Rep.. Ministry of Aviation; Royal Aircraft Establishment; RAE Farnborough.

Rowley, Clarence W, Colonius, Tim \& Basu, Amit J 2002 On self-sustained oscillations in two-dimensional compressible flow over rectangular cavities. Journal of Fluid Mechanics 455, 315-346.

Schmidt, Oliver T, Towne, Aaron, Colonius, Tim, Cavalieri, André VG, Jordan, Peter \& Brès, Guillaume A 2017 Wavepackets and trapped acoustic modes in a turbulent jet: coherent structure eduction and global stability. Journal of Fluid Mechanics 825, 1153-1181.

Siconolfi, Lorenzo, Citro, Vincenzo, Giannetti, Flavio, Camarri, Simone \& Luchini, PAOLO 2017 Towards a quantitative comparison between global and local stability analysis. Journal of Fluid Mechanics 819, 147-164.

TAm, Christopher KW \& Hu, FAng Q 1989a The instability and acoustic wave modes of supersonic mixing layers inside a rectangular channel. Journal of Fluid Mechanics 203, $51-76$. 
TAm, Christopher KW \& Hu, FAng Q $1989 b$ On the three families of instability waves of high-speed jets. Journal of Fluid Mechanics 201, 447-483.

Theofilis, Vassilios 2011 Global linear instability. Annual Review of Fluid Mechanics 43, 319-352.

Towne, Aaron, Cavalieri, André VG, Jordan, Peter, Colonius, Tim, Schmidt, Oliver, Jaunet, Vincent \& Brès, Guillaume A 2017 Acoustic resonance in the potential core of subsonic jets. Journal of Fluid Mechanics 825, 1113-1152.

Yamouni, S, Mettot, C, Sipp, D \& JacQuin, L 2013 Passive control of cavity flows. AerospaceLab (6), p-1.

Yu, Ming-Huei \& Monkewitz, Peter A 1990 The effect of nonuniform density on the absolute instability of two-dimensional inertial jets and wakes. Physics of Fluids A: Fluid Dynamics 2 (7), 1175-1181. 